\title{
ABSTRACT HOMOTOPY THEORY AND GENERALIZED SHEAF COHOMOLOGY
}

BY

\author{
KENNETH S. BROWN (1)
}

\begin{abstract}
Cohomology groups $H q(X, E)$ are defined, where $X$ is a topological space and $E$ is a sheaf on $X$ with values in Kan's category of spectra. These groups generalize the ordinary cohomology groups of $X$ with coefficients in an abelian sheaf, as well as the generalized cohomology of $X$ in the usual sense. The groups are defined by means of the "homotopical algebra" of Quillen applied to suitable categories of sheaves. The study of the homotopy category of sheaves of spectra requires an abstract homotopy theory more general than Quillen's, and this is developed in Part I of the paper. Finally, the basic cohomological properties are proved, including a spectral sequence which generalizes the Atiyah-Hirzebruch spectral sequence (in generalized cohomology theory) and the "local to global" spectral sequence (in sheaf cohomology theory).
\end{abstract}

Introduction. In this paper we will study the homotopy theory of sheaves of simplicial sets and sheaves of spectra. This homotopy theory will be used to give a derived functor definition of generalized sheaf cohomology groups $H^{q}(X, E)$, where $X$ is a topological space and $E$ is a sheaf of spectra on $X$, subject to certain finiteness conditions. These groups include as special cases the usual generalized cohomology of $X$ defined by a spectrum [22] and the cohomology of $X$ with coefficients in a complex of abelian sheaves. The cohomology groups have all the properties one would expect, the most important one be ing a spectral sequence

$$
E_{2}^{p q}=H^{p}\left(X, \pi_{-q} E\right) \Rightarrow H^{p+q}(X, E),
$$

which generalizes the Atiyah-Hirzebruch spectral sequence (in generalized cohomology) and the "local to global" spectral sequence (in sheaf cohomology), and

Received by the editors June 8, 1972 and, in revised form, October 15, 1972.

AMS (MOS) subject classifications (1970). Primary 55B20, 55B30, 55D99, 18F99; Secondary $55 \mathrm{~J} 10$.

Key words and phrases. Abstract homotopy theory, generalized sheaf cohomology, homotopical algebra, sheaf of spectra, homotopy category, derived functor.

(1) Partially supported by an NSF graduate fellowship and by NSF Grant GP28244. This paper is a revised version of the author's doctoral dissertation submitted to the Massachusetts Institute of Technology in May, 1971.

Copyright $\odot$ 1974, American Mathemation Society 
which yields a Leray spectral sequence for a map.

The cohomology the ory is developed in Part II of the paper. The necessary homotopy theory is done by showing that there are definitions of fibration, cofibration, and weak equivalence such that certain categories of sheaves satisfy Quillen's axioms for homotopy theory [21]. Because of the restrictions on the fategories of sheaves to which this applies, the resulting homotopy theory is somewhat unsatisfactory. For this reas on we develop in Part I (which is not essential for the main results of Part II) a more general abstract homotopy theory than that of [21], and we illustrate its application to sheaves in $\$ 3$.

I wish to thank my thesis advisor D. Quillen, who suggested the problem of defining generalized sheaf cohomology and who gave me helpful advice on how to attack the problem. The motivation for studying generalized sheaf cohomology was the hope that the theory might apply to (hi gher)algebraic K-theory. This application, together with some substantial improvements of the theory in case $X$ is a noetherian space of finite Krull dimension, has been worked out in collaboration with S. Gersten at the algebraic K-theory conference, at the Battelle Seattle Research Center (August 1972), and will appear in the proceedings of that conference.

\section{PART I. ABSTRACT HOMOTOPY THEORY}

1. Categories of fibrant objects. Let $\mathcal{C}$ be a category with finite products and a final object $e$. Assume that $\mathcal{C}$ has two distinguished classes of maps, called weak equivalences and fibrations. A map will be called an aspherical fibration if it is both a weak equivalence and a fibration. Following [21], we define a path space for an object $B$ to be an object $B^{I}$ together with maps

$$
B \stackrel{s}{\longrightarrow} B \stackrel{\left(d_{0}, d_{1}\right)}{\longrightarrow} B \times B,
$$

where $s$ is a weak equivalence, $\left(d_{0}, d_{1}\right)$ is a fibration, and the composite is the diagonal map. We will use the notation $\left(B^{I}, s, d_{0}, d_{1}\right)$ for a path space.

We will call $\mathcal{C}$ a category of fibrant objects for a bomotopy theory (or simply a category of fibrant objects) if the following axioms are satisfied.

(A) Let $f$ and $g$ be maps such that $g f$ is defined. If two of $f, g, g f$ are weak equivalences then so is the third. Any isomorphism is a weak equivalence.

(B) The composite of two fibrations is a fibration. Any isomorphism is a fibration.

(C) Given a diagram

$$
A \stackrel{u}{\longrightarrow} C \stackrel{v}{\longleftarrow} B,
$$


with $v$ a fibration, the fibred product $A \times{ }_{C} B$ exists and the projection pr: $A \times{ }_{C} B$ $\rightarrow A$ is a fibration. If $v$ is an aspherical fibration then so is pr. In other words, fibrations and aspherical fibrations are preserved by base extension.

(D) For any object $B$ there exists at least one path space $B^{I}$ (not necessarily functorial in $B$ ).

(E) For any object $B$ the map $B \rightarrow e$ is a fibration.

Examples. 1. If $\mathcal{C}^{\prime}$ is a model category [21] and if $\mathcal{C}$ is the full subcategory consisting of the fibrant objects in $C^{\prime}$ (i.e., the objects $B$ for which the map $B$ $\rightarrow e$ is a fibration), then $\mathcal{C}$ is a category of fibrant objects in the sense of the above definition.

2. Let $X$ be a topological space, let $\delta(X)$ be the category of sheaves on $X$ with values in the category of simplicial sets (or, equivalently, $\delta(X)$ is the category of simplicial objects in the category of sheaves of sets), and let $\delta(X)_{E}$ be the full subcategory consisting of those sheaves which stalkwise satisfy Kan's extension condition [11], [19]. We define a map to be a fibration (or weak equivalence) if it is stalkwise a fibration (or weak equivalence) in the sense of Kan. Then $\delta(X)_{E}$ is a category of fibrant objects.

3. If $\mathfrak{Q}$ is an abelian category then the category of chain complexes in $\mathfrak{Q}$ (infinite in both directions) is a category of fibrant objects, where a weak equivalence is defined as a map inducing homology isomorphisms and a fibration is a surjective map (cf. [13]).

The following lemma will be our basic tool in what follows.

Factorization lemma. If $\mathcal{C}$ is a category of fibrant objects and $u$ is any map in $\mathcal{C}$, then $u$ can be factored $u=p i$, where $p$ is a fibration and $i$ is right inverse to an aspherical fibration. (In particular, $i$ is a weak equivalence.)

Proof. We will imitate the standard method in (ordinary) homotopy theory for converting a map to a fibration. Let $u: A \rightarrow B$ be the given map, choose a path space $\left(B^{I}, s, d_{0}, d_{1}\right.$ ), and let $C=A \times_{B} B^{I}$ (which exists because $d_{0}: B^{I} \rightarrow B$ is an (aspherical) fibration-see [21]). Let $i: A \rightarrow C$ be the map with components $\left(\right.$ id $_{A}, s u$ ), and let $p: C \rightarrow B$ be the composite $d_{1}{ }^{\circ} \mathrm{pr}_{2}$. Then $i$ is right inverse to $\mathrm{Pr}_{1}$, which is an aspherical fibration, being a base extension of $d_{0}$; and $p$ is a fibration because it is the composite of

$$
A \times{ }_{B} B^{I} \stackrel{i \mathrm{~d} \times d_{1}}{\longrightarrow} A \times B
$$

with the projection $A \times B \rightarrow B$. (Note that id $\times d_{1}$ is the base extension of $B^{I}$ $\rightarrow B \times B$ by $u \times$ id: $A \times B \rightarrow B \times B$, and $A \times B \rightarrow B$ is a base extension of $A \rightarrow e$, so both are fibrations by axiom (C).) Then $u=p i$ is the required factorization. 
Remark. Notice that axiom (D) can be obtained from a weak form of the factorization lemma (which requires of $i$ only that it be a weak equivalence) applied to the diagonal map $B \rightarrow B \times B$. In some applications it is just as easy to verify directly this form of the factorization lemma as to verify axiom (D).

2. The homotopy category. Let $\mathcal{C}$ be any category with a distinguished class of maps called weak equivalences. Then by the bomotopy category of $\mathcal{C}$ (denoted Ho $\mathcal{C}$ ) we mean the category obtained from $\mathcal{C}$ by inverting the weak equivalences (see [11], [13], [21]).

In other words, we have a functor $\gamma: \mathcal{C} \rightarrow$ Ho $\mathcal{C}$ such that $\gamma(s)$ is an isomorphism if $s$ is a weak equivalence, and $\gamma$ is universal for this property.

The category Ho $C$ being defined by a universal property, it is a nontrivial matter to explicitly describe the maps in Ho $\mathcal{C}$. But in case $\mathcal{C}$ has the additional structure of a category of fibrant objects, which we now assume, we can obtain such a description by using an appropriate notion of homotopy, defined as follows. Two maps $f, g: A \rightrightarrows B$ are called bomotopic if for some path space $\left(B^{I}, s, d_{0}\right.$, $d_{1}$ ) there is a map $b: A \rightarrow B^{I}$ (called a bomotopy) such that $d_{0} b=f$ and $d_{1} b=g$. We will write $f \simeq g$. By piecing together path spaces as in [21] one can prove that homotopy is an equivalence relation.

As a first indication of the relevance of the concept of homotopy to the study of Ho $C$, note that homotopic maps become equal in Ho $\mathcal{C}$. In fact, since the two weak equivalences $d_{0}, d_{1}: B^{I} \rightrightarrows B$ have a common cross-section $s$,

$$
\gamma\left(d_{0}\right)=\gamma(s)^{-1}=\gamma\left(d_{1}\right),
$$

which implies $\dot{\gamma}(f)=\gamma(g)$.

The behavior of the homotopy relation with respect to composition of maps is described in Proposition 1 below.

Lemma 1. Any diagram

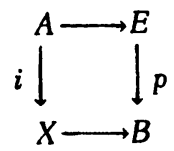

with $i$ a weak equivalence and $p$ a fibration can be imbedded in a diagram

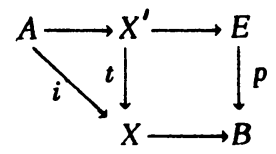

with $t$ an aspherical fibration.

Proof. Apply the factorization lemma to the map $A \rightarrow X \times_{B} E$. 
Lemma 2. Let $u: B \rightarrow C$ be a map and let $\left(B^{I}, s, d_{0^{\prime}} d_{1}\right)$ and $\left(C^{I}, s^{C}, d_{0}^{C}\right.$, $\left.d_{1}^{C}\right)$ be path spaces. Then we can find a path space $\left(B^{I^{\prime}}, s^{\prime}, d_{0^{\prime}}^{\prime} d_{1}^{\prime}\right)$, an aspherical fibration $t: B^{I^{\prime}} \rightarrow B^{I}$, and a map $\bar{u}: B^{I^{\prime}} \rightarrow C^{I}$ such that the following diagram commutes:

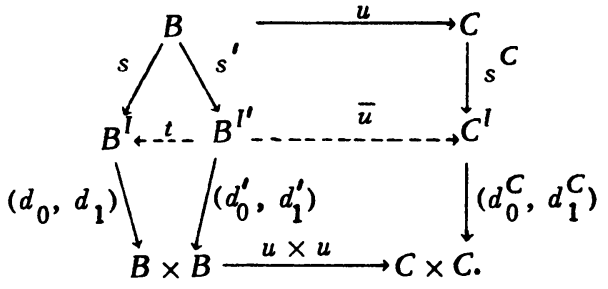

Proof. This is an easy consequence of Lemma 1 applied to the square

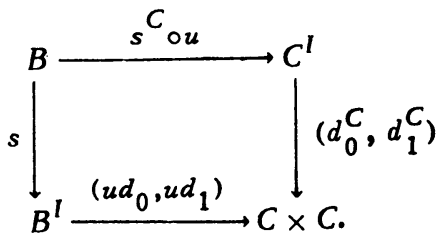

Proposition 1. Assume $f \simeq g: A \rightarrow B$. Then

(i) if $u: C \rightarrow A$ is arbitrary then $f u \simeq g u: C \rightarrow B$;

(ii) if $u: B \rightarrow C$ is arbitrary and $C^{I}$ is any path space for $C$, then there is an aspherical fibration $t: A^{\prime} \rightarrow A$ such that uft $\simeq$ ugt: $A^{\prime} \rightarrow C$ by a bomotopy b: $A^{\prime} \rightarrow C^{I}$.

Proof. (i) is trivial. For (ii), let $b: A \rightarrow B^{I}$ be a homotopy from $f$ to $g$, let $C^{I}$ be an arbitrary path space for $C$, and let $B^{I^{\prime}}$ be as in Lemma 2. Then we can take

$$
A^{\prime}=A \times{ }_{B} B^{I^{\prime}}
$$

$t=\mathrm{pr}: A^{\prime} \rightarrow A$, and then the composite

$$
A^{\prime} \stackrel{\mathrm{pr}}{\longrightarrow} B^{I^{\prime}} \stackrel{\bar{u}}{\longrightarrow} C^{I}
$$

is a homotopy from uft to ugt.

We now define, as an approximation to Ho $\mathcal{C}$, a category ${ }_{\mathfrak{C}} \mathcal{C}$ with the same objects as $\mathcal{C}$ and with $\operatorname{Hom}_{\pi \mathcal{C}}(A, B)$ equal to the quotient of $\operatorname{Hom}_{\mathcal{C}}(A, B)$ by the equivalence relation $f \sim g$ if there is a weak equivalence $t: A^{\prime} \rightarrow A$ such that $f t \simeq g t$. [The proof that this relation is transitive makes use of Proposition 2(a) below as well as Proposition 1. The same is true of the proof that the relation is compatible with composition of maps.] It is clear that Ho $\mathcal{C}$ is obtained from 
${ }_{\pi} \mathcal{C}$ by inverting the weak equivalences, and this localization is easily described because of the following proposition.

Proposition 2. The class of weak equivalences in $\pi \mathcal{C}$ admits a calculus of right fractions in the sense of [11], i.e.,

(a) any diagram

$$
A \rightarrow C \stackrel{t}{\leftarrow} B
$$

in $\mathcal{C}$ with $t$ a weak equivalence can be imbedded in a bomotopy commutative square<smiles>[Y]1[CH]B[Te]1</smiles>

witb $t^{\prime}$ a weak equivalence; and

(b) given $f, g: A \rightrightarrows B$, if there is a weak equivalence $t: B \rightarrow C$ sucb that $t f \simeq t g$, then there is a weak equivalence $t^{\prime}: A^{\prime} \rightarrow A$ sucb that $f t^{\prime} \simeq g t^{\prime}:$

$$
A^{\prime}-t^{\prime}-\rightarrow A \underset{\mathrm{g}}{\stackrel{f}{\rightrightarrows}} B \stackrel{t}{\longrightarrow} C .
$$

The proof is based on the "homotopy-theoretic fibred product":

Lemma 3. Given a diagram

$$
A \stackrel{u}{\rightarrow} C \stackrel{v}{\longleftarrow} B,
$$

the projection $A \times{ }_{C} C^{I} \times_{C} B \rightarrow A$ is a fibration. Furthermore it is aspherical if $v$ is a weak equivalence.

Proof. The projection is the base extension by $u$ of a map $C^{I} \times{ }_{C} B \rightarrow C$ which is essentially the same as the fibration $p$ constructed in the proof of the factorization lemma. The result is now immediate.

Proof of Proposition 2. Property (a) follows from the lemma by taking $A^{\prime}=$ $A \times{ }_{C} C^{I} \times{ }_{C} B$ and $t^{\prime}=\mathrm{pr}: A^{\prime} \rightarrow A$.

To prove (b), let $b: A \rightarrow C^{I}$ be a homotopy from $t f$ to $t g$, where $\left(C^{I}, s, d_{0}\right.$, $\left.d_{1}\right)$ is a path space, let $D=B \times{ }_{C} C^{I} \times{ }_{C} B$, and observe that we have a map $H$ : $A \rightarrow D$ whose components are $(f, h, g)$ and a map $i: B \rightarrow D$ whose components are (id, st, id). Now the projections $D \rightrightarrows B$ are weak equivalences by the lemma, and it follows that $i$ is a weak equivalence. Thus if we factor $i$ as a composite $B \rightarrow D^{\prime} \rightarrow D$ as in the factorization lemma, the map $D^{\prime} \rightarrow D$ will be an aspherical fibration. On the other hand, the projection $D \rightarrow B \times B$ is a fibration, being a base extension of $C^{I} \rightarrow C \times C$, and so the composite $D^{\prime} \rightarrow D \rightarrow B \times B$ is a 
fibration and $D^{\prime}$ is a path space for $B$. We now let $A^{\prime}=A \times_{D} D^{\prime}$ and $t^{\prime}=\mathrm{pr}$ : $A^{\prime} \rightarrow A$, and then pr: $A^{\prime} \rightarrow D^{\prime}$ is a homotopy from $f t^{\prime}$ to $g t^{\prime}$, which completes the proof.

If $A$ and $B$ are objects of $\mathcal{C}$, let

$$
[A, B]=\underline{\lim } \operatorname{Hom}_{\pi \mathrm{e}}\left(A^{\prime}, B\right),
$$

where the (filtering) index category for the direct limit is the category in which an object is a weak equivalence $[t]: A^{\prime} \rightarrow A$ in ${ }_{\pi} \mathcal{C}$ and a map from $[t]: A^{\prime} \rightarrow A$ to $\left[t^{\prime}\right]: A^{\prime \prime} \rightarrow A$ is a commutative triangle in ${ }^{\infty} \mathcal{C}$

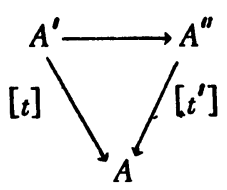

From [11] and Proposition 2 we obtain the following theorem.

Theorem 1. If $\mathcal{C}$ is a category of fibrant objects and $A$ and $B$ are any two objects of $\mathcal{C}$, then there is a canonical isomorphism

$$
\operatorname{Hom}_{\mathrm{Ho}} \mathrm{e}^{(A, B)} \approx[A, B] .
$$

In particular, if $\gamma: \mathcal{C} \rightarrow \mathrm{Ho} \mathcal{C}$ is the localization functor, then

(i) any map in Ho $C$ can be written as a "right fraction" $\gamma(f) y(t)^{-1}$, where $t$ is a weak equivalence; and

(ii) if $f, g: A \Rightarrow B$ are maps in $C$ then $\gamma(f)=\gamma(g)$ if and only if there is a weak equivalence $t: A^{\prime} \rightarrow A$ sucb that $f t \simeq g t$.

Remarks. 1. In many applications of abstract homotopy theory the category of fibrant objects $\mathcal{C}$ is a full subcategory of a category $\mathcal{C}^{\prime}$, and it is really Ho $C^{\prime}$ that we are interested in. For an example of how Theorem 1 can be used to describe Ho $\mathcal{C}^{\prime}$, see $\$ 8$ below (in particular, Proposition 7 and Corollary 3 ).

2. It is sometimes convenient to use a slight modification of Theorem 1 obtained by replacing "weak equivalence" by "aspherical fibration". The proof remains valid (and even simplifies) with this modification. [One needs to observe that the category obtained from $\mathcal{C}$ by inverting the aspherical fibrations coincides with $\mathrm{Ho} \mathcal{C}$, because of the factorization lemma.]

3. An illustration: Verdier's hypercovering theorem. This section is intended both to illustrate Theorem 1 by giving an interesting application and to introduce some concepts and results concerning simplicial sheaves which we will later need in the somewhat less familiar setting of sheaves of spectra.

Let $X$ be a topological space and let $S(X)_{E}$ be as in Example 2 of $\$ 1$. We 
will also consider the category $\mathcal{S}_{a b}(X)$ of simplicial abelian sheaves on $X$ (i.e., simplicial objects in the category of sheaves of abelian groups), the category $\mathcal{C}(x)$ of complexes of abelian sheaves (indexed with superscripts, with differential of degree +1$)$, and the full subcategory $\mathcal{C}-(X)$ of $\mathcal{C}(X)$ consisting of the complexes which are zero in positive dimensions.' Note that $\mathcal{S}_{a b}(X)$ inherits a notion of weak equivalence from $S(X)_{E}$ and that $\mathcal{C}(X)$ and $\mathcal{C}-(X)$ have an obvious notion of weak equivalence (map inducing homology isomorphisms). Thus (see the beginning of $\S 2$ ) we have categories $H o \delta(X)_{E}$, Ho $\delta_{a b}(X)$, Ho $\mathcal{C}(X)$, and Ho $\mathcal{C}-(X)$. To avoid cumbersome notation, we will throughout the remainder of this section denote these four homotopy categories by $\mathscr{D}_{E}, \mathfrak{D}_{\text {ab }}, \mathfrak{D}_{\text {, and }} \mathfrak{D}^{-}$.

Adjoint functor lemma. Let $S: \mathcal{C}_{1} \rightarrow \mathcal{C}_{2}$ be left adjoint to $T: \mathcal{C}_{2} \rightarrow \mathcal{C}_{1}$, where $C_{1}$ and $C_{2}$ are categories with a notion of weak equivalence. If $S$ and $T$ preserve weak equivalences, then the induced functors Ho $S:$ Ho $C_{1} \rightarrow \mathrm{Ho}_{2}$ and Ho $T: \mathrm{Ho}_{2} \rightarrow \mathrm{Ho} \mathcal{C}_{1}$ are adjoint.

Proof. The adjunction maps $a: S T \rightarrow I d C_{2}$ and $\beta: I d C_{1} \rightarrow T S$ induce corresponding natural transformations in the homotopy categories (cf. [21, I, top of p. 4.3]). Since the composites $T \stackrel{\beta T}{\longrightarrow} T S T \stackrel{T a}{\longrightarrow} T$ and $S \stackrel{S \beta}{\longrightarrow} S T S \stackrel{a S}{\longrightarrow} S$ are identity maps in $\mathcal{C}_{1}$ and $\mathcal{C}_{2}$, the analogous maps in the homotopy categories are also identities, which proves Ho $S$ left adjoint to Ho $T$.

The hypercovering theorem (below) will result from combining the adjoint functor lemma with Theorem 1, after we make some elementary observations:

(1) The functor $\mathfrak{D}^{-} \rightarrow \mathfrak{D}$ induced by the inclusion $s: \mathcal{C}(x) \rightarrow \mathcal{C}(x)$ is fully faithful. [In fact, $S$ has a right adjoint $T$ which preserves weak equivalences, so Ho $S$ is left adjoint to Ho $T$. Now since $S$ is fully faithful, the adjunction map $\beta: l d \rightarrow T S$ is an isomorphism. But then the same is true of the analogous adjunction map in $D^{-}$, and this implies that Ho $S$ is fully faithful. (See [13] for another proof, based on a special case of Theorem 1.)]

(2) For any abelian sheaf $F$ and integer $q \geq 0, H^{q}(X, F)=\left[Z, F_{(q)}\right]_{\mathscr{D}}$, where $\mathrm{Z}$ is the complex concentrated in dimension zero consisting of the constant sheaf with stalk equal to the group of integers, and $F_{(q)}$ is $F$ concentrated in dimension $-q$. [This follows from Theorem 1 and the derived functor definition of $H^{q}(X, F)$. The point is that if we replace $F_{(q)}$ by a bounded below complex $I^{\circ}$ of injectives, the direct system defining $\left[\mathrm{Z}, I^{\circ}\right]$ in Theorem 1 is constant (cf. [13] or [21]).]

(3) The normalized chain complex functor $N$ : $\mathcal{S}_{a b}(X) \rightarrow \mathcal{C}-(X)$ is an equivalence of categories which preserves weak equivalences and the usual notions of homotopy (simplicial (abelian) homotopy and chain homotopy) [9, §3]. We will denote by $K(F, q)$ the object of $\int_{a b}(X)$ corresponding to the complex $F_{(q)}$ of (2). 
(4) The forgetful functor $\mathcal{S}_{a b}(X) \rightarrow \mathcal{S}(X)_{E}$ is right adjoint to a functor $\mathbf{Z}$ : $\mathcal{S}(X)_{E} \rightarrow \mathcal{S}_{a b}(X)$, the "free abelian sheaf" functor, which preserves weak equivalences. [The fact that $\mathrm{Z}$ preserves weak equivalences follows from the relative Hurewicz theorem, but here is a short proof more in the spirit of this paper: The question being a stalkwise one, we may assume $X$ is a point. The two categories can then be given the structure of closed model category (see [21] and the remark in $\$ 6$ below), and by the dual of the factorization lemma ( $\$ 1)$, it suffices to check that $\mathrm{Z}$ preserves the injective weak equivalences. Now these maps can be characterized by lifting properties [21], and the result follows by "adjoint functor chas ing" from the fact that the forgetful functor preserves fibrations.] If $e$ denotes the final object of $S(X)_{E}$ (the sheaf which stalkwise has only one simplex in each dimension), then $\mathrm{Z} e$ is the simplicial abelian sheaf which corresponds under the equivalence of (3) to the complex $Z$ of (2).

(5) Theorem 1, applied to the category $S(X)_{E}$, remains valid if $\operatorname{Hom}_{\pi \mathcal{C}}(\cdot, \cdot)$ is replaced by $\pi(\cdot, \cdot)$, which by definition consists of simplicial homotopy classes of maps. [In fact, the definition of simplicial homotopy is based on a particular choice of path space $B^{I}$ (see [11] or [19]), and Proposition 1 (ii) of $\$ 2$, applied to id: $B \rightarrow B$, shows that, as far as the direct limit of Theorem 1 is concerned, it does not matter which path space we use.] We will use the same symbol $\pi(\bullet,$, in the proof below to denote chain homotopy classes of maps, and we will use $\pi_{\mathrm{ab}}$ to denote simplicial abelian homotopy classes.

Theorem 2 (Verdier [2, Exposé V, Appendix], [3]). If $F$ is an abelian sheaf, then

$$
H^{q}(X, F) \approx \frac{\lim }{K} H^{q}\left(C^{\cdot}(K, F)\right),
$$

where $K$ ranges over the bypercoverings of $X$ (i.e., the objects of $S(X)_{E}$ such that the map $K \rightarrow e$ is a weak equivalence) and where $C^{\circ}(K, F)$ is the complex of abelian groups associated to the cosimplicial abelian group obtained from $K$ by applying the functor $\operatorname{Hom}_{\text {(sheares) }}(\cdot, F)$ dimension-wise.

Proof. We first express sheaf cohomology in terms of Eilenberg-Mac Lane sheaves:

$$
\begin{array}{rlr}
H^{q}(X, F) & =[\mathrm{Z}, F(q)]_{\mathcal{D}} & \text { by (2) } \\
& =[\mathrm{Z}, F(q)]_{\mathcal{D}^{-}} & \text {by (1) } \\
& =[\mathrm{Z} e, K(F, q)]_{\mathscr{D}_{\mathrm{ab}}} & \text { by (3) } \\
& =[e, K(F, q)]_{\mathscr{D}_{E}} \quad \text { by (4) and the adjoint functor lemma. }
\end{array}
$$


Now we use (5) to rewrite the last group as $\lim _{\rightarrow} \pi(K, K(F, q))$, and the proof will be complete once we make the standard indentification of cohomology classes with maps into Eilenberg-Nac Lane complexes. In fact,

$$
\begin{aligned}
\pi(K, K(F, q)) & =\pi_{\mathrm{ab}}(\mathrm{Z} K, K(F, q)) & & \text { by (4) } \\
& \left.=\pi^{(N \mathrm{Z} K, F(a)}\right) & & \text { by (3) } \\
& =H^{q}\left(C^{\cdot}(K, F)\right) & & \text { by inspection. }
\end{aligned}
$$

4. The loop functor and fibration sequences. Let $\mathcal{C}$ be a category of fibrant objects. For any object $B$ of $\mathcal{C}$, let $\mathcal{C}_{B}$ denote the category of fibrations over $B$. If we define fibration and weak equivalence in $\mathcal{C}_{B}$ by means of the forgetful functor $\mathcal{C}_{B} \rightarrow \mathcal{C}$, it is trivial to check that $\mathcal{C}_{B}$ is also a category of fibrant objects. [Use the factorization lemma $(\$ 1)$ and the remark following it.]

Lemma 1. If $u: B^{\prime} \rightarrow B$ is a map in $\mathcal{C}$, then the base change functor $u^{*}:$ $\mathcal{C}_{B} \rightarrow \mathcal{C}_{B^{\prime}}$ preserves fibrations and weak equivalences.

Proof. According to the factorization lemma of $\$ 1$ (applied to the category $\mathcal{C}_{B}$ ), it is sufficient to prove that $u^{*}$ preserves fibrations and aspherical fibrations. This is easy once one observes that if $E_{1} \rightarrow E_{2}$ is a fibration of fibre spaces over $B$, then

$$
B^{\prime} \times{ }_{B} E_{1}=\left(B^{\prime} \times{ }_{B} E_{2}\right) \times_{E_{2}} E_{1} \text {. }
$$

Lemma 2. The base extension of a weak equivalence in $\mathcal{C}$ by a fibration is a weak equivalence.

Proof. Let $p: E \rightarrow B$ be the fibration and $u: B^{\prime} \rightarrow B$ the weak equivalence. We must prove that pr: $B^{\prime} \times_{B} E \rightarrow E$ is a weak equivalence. By the factorization lemma we may assume that $u$ is right inverse to an aspherical fibration $v$ : $B \rightarrow B^{\prime}$. Let $E_{1}=B \times_{B^{\prime}} E$ be the fibred product of $v$ and $v p$. Then the map $f=\left(p\right.$, id): $E \rightarrow E_{1}$ is a map in $C_{B}$ and is a weak equivalence because it is right inverse to the aspherical fibration (in $\mathcal{C}$ ) $\mathrm{pr}: E_{1} \rightarrow E$. Thus (using Lemma 1) we see that the horizontal maps in the following diagram are weak equivalences:

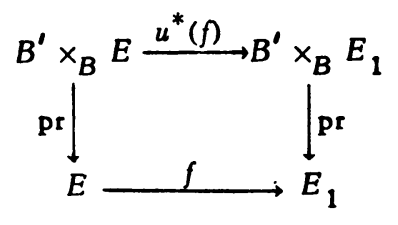

We are now reduced to proving the lemma for $E_{1}$ instead of $E$, i.e., we need only show that the right hand vertical map is a weak equivalence. But this is 
immediate, because $B^{\prime} \times_{B} E_{1}=B^{\prime} \times_{B}\left(B \times_{B^{\prime}} E\right)=E$ and $\mathrm{pr}: B^{\prime} \times_{B} E_{1} \rightarrow E_{1}$ is right inverse to the aspherical fibration $\mathrm{pr}: E_{1} \rightarrow E$.

Lemma 3. Assume $\mathcal{C}$ is pointed (i.e., the final object $e$ is also initial) and let

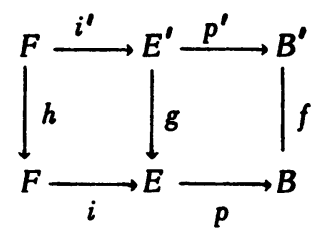

be a commutative diagram in which $p$ and $p^{\prime}$ are fibrations and $i$ and $i^{\prime}$ are the fibre inclusions (i.e., $F=e \times_{B} E$ and $F^{\prime}=e \times_{B^{\prime}} E^{\prime}$ ). If $f$ and $g$ are weak equive alences then so is $b$.

Proof. Let $E^{\prime \prime}=B^{\prime} \times{ }_{B} E$, so that we have a diagram

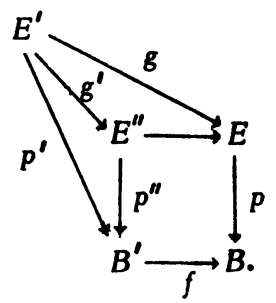

The unlabelled arrow is a weak equivalence by Lemma 2, and thus $g^{\prime}$ is a weak equivalence. It now follows from Lemma 1 that the induced map of $F^{\prime}$ to the fibre of $p^{n}$ is a weak equivalence. But the fibre of $p^{n}$ is $F$ and the induced map $F^{\prime} \rightarrow F$ is $h$, which completes the proof.

Lemma 4. Assume $C$ is pointed. Let $p_{1}: E_{1} \rightarrow B$ and $p_{2}: E_{2} \rightarrow C$ be fibrations with fibres $F_{1}, F_{2}$, let $u: B \rightarrow C$ be a map, let f, g: $E_{1} \rightrightarrows E_{2}$ be maps such that $p_{2} f=p_{2} g=u p_{1}$, and let $t: E_{1}^{\prime} \rightarrow E_{1}$ be a weak equivalence such that $f t=g t$ :

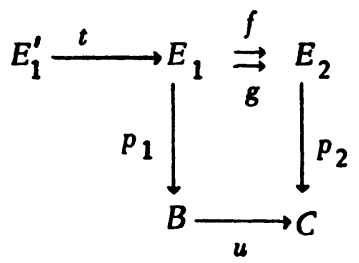

Then the maps $F_{1} \Rightarrow F_{2}$ induced by $f$ and $g$ coincide ir Ho $\mathcal{C}$.

Proof. The hypotheses and conclusion remain unchanged if we replace $p_{2}$ by pr: $B \times{ }_{C} E_{2} \rightarrow B$, so we may assume $C=B$ and $u=$ id. Let $\left(X, s, d_{0}, d_{1}\right)$ 
be a path space for $E_{2}$ in $C_{B}$. By applying Lemma 1 of $\$ 2$ to the'square

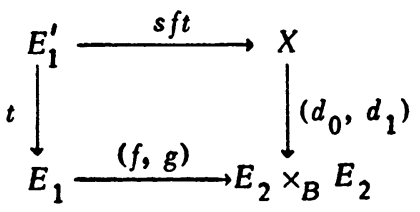

we obtain a square

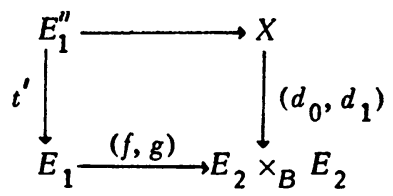

where $t^{\prime}$ is an aspherical fibration. Then $E_{1}^{\prime \prime}$ is a fibre space over $B$ and this square can be regarded as a square in $\mathcal{C}_{B}$. It is clear from this that $f$ and $g$ become equal in $\mathrm{Ho}_{B} \mathcal{C}_{B}$, and the result now follows from the fact that (by Lemma 1) there is a commutative diagram of categories and functors (induced by the fibre functor $\mathcal{C}_{B} \rightarrow \mathcal{C}$ )

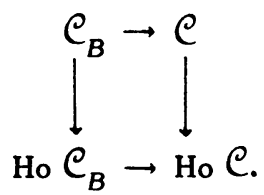

Theorem 3. Let $\mathcal{C}$ be a pointed category of fibrant objects. Then there is a functor $\Omega: \mathrm{Ho} C \rightarrow \mathrm{Ho} C$ such that for any object $B$ and any path space $B^{I}$. $\Omega B$ can be canonically identified with the fibre of $B^{I} \rightarrow B \times B$. Furthermore, $\Omega B$ bas a natural group structure.

Proof. For any path space $B^{I}$ let $\Omega^{(I)} B$ be the fibre of $B^{I} \rightarrow B \times B$. I claim that if $B^{I^{\prime}}$ is another path space, $\Omega^{(I)} B$ and $\Omega^{\left(I^{\prime}\right)} B$ are canonically isomorphic in Ho $C$. In fact, if there is a map $B^{I} \rightarrow B^{I^{\prime}}$ compatible with all the maps occurring in the definition of path space, any such map induces a weak equivalence $\Omega^{(I)_{B}} \rightarrow \Omega^{\left(I^{\prime}\right)_{B}}$ (by Lemma 1 ), and any two such maps of path spaces induce the same map $\Omega^{(I)} B \rightarrow \Omega^{\left(I^{\prime}\right)_{B}}$ in Ho $C$ by Lemma 4 applied to the diagram

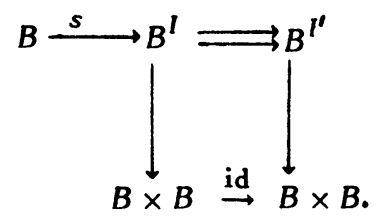

This proves the assertion in a special case, and the general case follows from the fact that for any two path spaces there is a third which maps to both of them 
(Lemma 2 of $\S 2$ ). We can now write $\Omega B$ instead of $\Omega^{(l)} B$, where we regard $\Omega B$ as an object of $\mathrm{Ho} C$ well-defined up to canonical isomorphism. By arguments similar to those just given, we can associate to a map $B \rightarrow C$ in $\mathcal{C}$ a map $\Omega B \rightarrow \Omega C$ in Ho $\mathcal{C}$, so that we have a functor $\Omega: \mathcal{C} \rightarrow$ Ho $\mathcal{C}$. By Lemma 3 and the fact that products preserve weak equivalences (which is essentially a special case of Lemma 1), this functor preserves weak equivalences and so can be regarded as a functor $\Omega: \mathrm{Ho} C \rightarrow$ Ho $\mathcal{C}$.

To give $\Omega B$ a group structure, let $B^{I}$ and $B^{I^{\prime}}$ be any two path spaces and let $B^{I+I^{\prime}}=B^{I} \times_{B} B^{I^{\prime}}$. Then there is an obvious map $\Omega^{(I)} B \times \Omega^{\left(I^{\prime}\right)_{B}} \rightarrow \Omega^{\left(I+I^{\prime}\right)_{B}}$ which gives us a product $m: \Omega B \times \Omega B \rightarrow \Omega B$ in Ho $C$. The product is easily seen to be well defined and associative. The fact that $e$ is an identity for this multiplication is immediate from the definitions and from the fact that $\left(s d_{0}\right.$, id): $B^{I} \rightarrow B^{I} \times{ }_{B} B^{I}$ and (id, $s d_{1}$ ): $B^{I} \rightarrow B^{I} \times_{B} B^{I}$ are maps of path spaces. Finally, the inverse $\Omega B \rightarrow \Omega B$ is induced by id: $\Omega^{(I)} B \rightarrow \Omega^{\left(I^{*}\right)} B$, where $\Omega^{(I)} B$ corresponds to ( $B^{I}$, $\left.s, d_{0}, d_{1}\right)$ and $\Omega^{\left(I^{*}\right)} B$ corresponds to $\left(B^{I}, s, d_{1}, d_{0}\right)$. To see that this is actually a (right) inverse, it is clear from the definitions that we need only show that the two maps $\Omega^{(I)} B \rightrightarrows \Omega^{\left(I+l^{*}\right)} B$ induced by

$$
B^{I} \underset{\left(s d_{0}, s d_{0}\right)}{\stackrel{\text { diag }}{\longrightarrow}} B^{I} \times_{B} B^{I}
$$

coincide in Ho $C$, where $B^{I} \times B B^{I}=B^{I+I^{*}}$ is the fibred product of $d_{1}$ with itself. This follows from Lemma 4 applied to

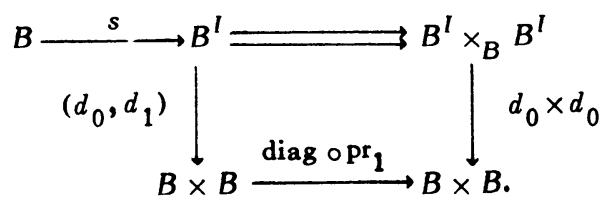

Proposition 3. Let $\mathcal{C}$ be a pointed category of fibrant objects and let $p: E$ $\rightarrow B$ be a fibration with fibre $F$. Then there is a natural map $a: F \times \Omega B \rightarrow F$ in Ho $C$ which defines a right action of the group $\Omega B$ on $F$.

Proof. As in $[21, I, \S 3]$ we construct path spaces $E^{I}, B^{I}$ related by a fibration $E^{I} \rightarrow E \times_{B} B^{I} \times{ }_{B} E$, and we then deduce an aspherical fibration $E^{I} \rightarrow E \times_{B} B^{I}$. By base extension we get an aspherical fibration $t: F \times{ }_{E} E^{I} \times{ }_{E} F \rightarrow F \times \Omega B$, and the desired map is then $\mathrm{pr}_{3} t^{-1}$. It is straightforward, using the techniques we have developed, to verify that this is well defined and has the desired properties.

We now compute this action in a special case. Let $A \rightarrow B$ be a map in $\mathcal{C}$, and let $A \rightarrow E \rightarrow B$ be a factorization as constructed in the proof of the factorization lemma of $\$ 1$, i.e., $E=A \times_{B} B^{I}$. Let $B^{2 I}=B^{I} \times{ }_{B} B^{I}$ and let $E^{\prime}=A \times_{B} B^{2 I}$. 
Notice that we have a map of path spaces

$$
B^{I} \stackrel{\left(\text { id, } s d_{1}\right)}{\longrightarrow} B^{I} \times_{B} B^{I}=B^{2 I}
$$

which induces by base extension a weak equivalence $w: E \rightarrow E^{\prime}$. Regarding $E$ and $E^{\prime}$ as fibre spaces over $B$ with fibres $F, F^{\prime}$, we obtain a weak equivalence $\bar{w}: F \rightarrow F^{\prime}$. On the other hand, we have an obvious map (in fact, an isomorphism)

$$
f: E \times_{B} B^{I} \rightarrow E^{\prime},
$$

which we regard as a map of a fibre space over $B \times B$ to a fibre space over $B$, lying over $\mathrm{pr}_{2}: B \times B \rightarrow B$. The induced map on the fibres is a map $F: F \times \Omega B$ $\rightarrow F^{\prime}$, and I claim that the composite $\bar{w}^{-1} \circ \bar{f}$ in $\mathrm{Ho} C$ is the action $F \times \Omega B \rightarrow F$. We prove this as follows. Construct a path space $E^{I}$ as in the proof of Proposition 3 and consider the two maps $E^{I} \rightrightarrows E^{\prime}$ obtained by going around the square

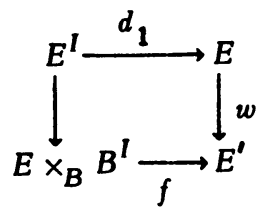

in the two possible ways. By.applying Lemma 4 to the diagram

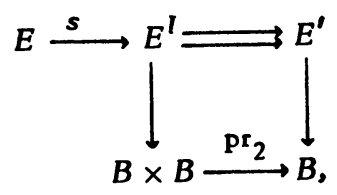

we deduce from $\left(^{*}\right)$ a commutative square in Ho $C$

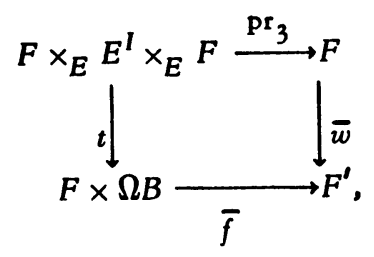

which, in view of the proof of Proposition 3, is exactly what is needed to prove our assertion.

Following [21], we now define a fibration sequence to be a diagram $F \rightarrow E$ $\rightarrow B$ in Ho $\mathcal{C}$ together with an action $F \times \Omega B \rightarrow F$ in $\mathrm{Ho} \mathcal{C}$ which are isomorphic to the diagram and action obtained from a fibration in $\mathcal{C}$.

Proposition 4. Given a fibration sequence

$$
F \stackrel{i}{\rightarrow} E \stackrel{p}{\rightarrow} B, \quad F \times \Omega B \stackrel{a}{\rightarrow} F,
$$


let $i^{\prime}$ be the composite

$$
\Omega B \stackrel{(e, j)}{\longrightarrow} F \times \Omega B \stackrel{a}{\rightarrow} F
$$

where $j: \Omega B \rightarrow \Omega B$ is the group inverse, and let $a^{\prime}$ be the composite

$$
\Omega B \times \Omega E \stackrel{\text { id } \times \Omega_{p}}{\longrightarrow} \Omega B \times \Omega B \stackrel{m}{\rightarrow} \Omega B .
$$

Then

$$
\Omega B \stackrel{i^{\prime}}{\rightarrow} F \stackrel{i}{\rightarrow} E, \quad \Omega B \times \Omega E \stackrel{a^{\prime}}{\rightarrow} \Omega B
$$

is a fibration sequence.

Proof. We may assume that $p$ is a fibration in $\mathcal{C}$ with fibre $F$ and that $a$ is the action constructed in the proof of Proposition 3. If we convert $i$ into a fibration $p^{\prime}$ as in the proof of the factorization lemma, the fibre of that fibration, $F \times{ }_{E} E^{l} \times_{E} e$, admits an aspherical fibration to $\Omega B$, namely the base extension of $E^{I} \rightarrow B^{I} \times_{B} E$ by

$$
\Omega B \stackrel{(k, e)}{\longrightarrow} B^{l} \times_{B} E,
$$

where $k$ is the inclusion of $\Omega B$ into $B^{I}$. We thus identify this fibre with $\Omega B$ in Ho $C$, and, using the aspherical fibration pr: $F \times_{E} E^{I} \rightarrow F$ to identify $F$ with the total space of $p^{\prime}$, it is immediate that the fibre inclusion becomes identified with $i^{\prime}$. The fact that the action is given by $a^{\prime}$ follows from the discussion following Proposition 3.

Corollary 1. Under the bypotheses of Proposition 4, there is an exact sequence in Ho $\mathcal{C}$

$$
\cdots \rightarrow \Omega E \rightarrow \Omega B \rightarrow F \rightarrow E \rightarrow B,
$$

where exactness is interpreted as in $[21,1, p .3 .8]$.

The proof is straightforward, using Theorem $1(\$ 2)$. Note that the homotopy lifting property which one usually uses in this context still holds when one passes to the direct limit, in view of the fact that $E^{I} \rightarrow E \times_{B} B^{I}$ is an aspherical fibration.

Corollary 2 Let $\mathcal{C}$ be a pointed category of fibrant objects and let $P \rightarrow B$ be a fibration with fibre $F$, where $P$ is weakly equivalent to $e$. Then $F$ is canonically isomorpbic to $\Omega B$ in $\mathrm{Ho} \mathcal{C}$.

Proof. Exactness of $e \rightarrow \Omega B \rightarrow F \rightarrow e$ means that the group $\Omega B$ acts 
"transitively and without fixed points" on $F$, so $\Omega B \rightarrow F$ is an isomorphism.

Remark. If we apply the discussion following Proposition 3 to the diagonal map of $B$, then we see easily that the natural action of $\Omega B \times \Omega B$ on $\Omega B$ induced by any path fibration $B^{I} \rightarrow B \times B$ is given by two-sided translation (i.e., $\left.g \cdot\left(g^{\prime}, g^{\prime \prime}\right)=g^{\prime-1} g g^{\prime \prime}\right)$. This is useful in the following situation. Given two maps from $A$ to $B$, we form the "equalizer"

$$
K=A \times_{B \times B} B^{I}
$$

and we have a fibration sequence in Ho $\mathcal{C}$

$$
\cdots \rightarrow \Omega K \rightarrow \Omega A \stackrel{\beta}{\rightarrow} \Omega B \rightarrow K \rightarrow A,
$$

where, because of the above description of the action of $\Omega B \times \Omega B$ on $\Omega B$, we can identify $\beta$ as the "difference" of the two maps from $\Omega A$ to $\Omega B$.

5. A theorem on inverse limits. The purpose of this section is to prove, in our abstract setting, a well-known theorem on the inverse limit of a tower of fibrations. The theorem will not be needed in Part II of this paper. We introduce, for any category of fibrant objects $\mathcal{C}$, the category Tow $(\mathcal{C})$ of diagrams

$$
\cdots \rightarrow A_{i} \stackrel{p_{i}}{\rightarrow} A_{i-1} \rightarrow \cdots
$$

where each $p_{i}$ is a fibration in $\mathcal{C}$ and where $A_{i}=e$ for sufficiently small $i$. A map of towers $\left\{A_{i}\right\} \rightarrow\left\{B_{i}\right\}$ will be called a weak equivalence if each map $A_{i} \rightarrow$ $B_{i}$ is a weak equivalence in $C_{\text {; }}$ the map will be called a fibration if each map

$$
A_{i} \rightarrow A_{i-1} \times_{B_{i-1}} B_{i}
$$

is a fibration in $\mathcal{C}$. It is easy to verify that with these definitions Tow $(\mathcal{C})$ is a category of fibrant objects. For example, to verify the first part of axiom (C), given

$$
\left\{\dot{A}_{i}\right\} \stackrel{u}{\rightarrow}\left\{C_{i}\right\} \stackrel{v}{\leftarrow}\left\{B_{i}\right\}
$$

with $v$ a fibration, let

$$
D_{i}=A_{i} \times_{C_{i}} B_{i}
$$

and we must show that pr: $\left\{D_{i}\right\} \rightarrow\left\{A_{i}\right\}$ is a fibration (which will also imply that the maps $D_{i} \rightarrow D_{i-1}$ are fibrations, so that $\left\{D_{i}\right\}$ is an object of Tow $(\mathcal{C})$ ). In other words, the maps

$$
D_{i} \rightarrow D_{i-1} \times_{A_{i-1}} A_{i}
$$


must be fibrations in $\mathcal{C}$. But

$$
D_{i-1} \times A_{i-1} A_{i}=A_{i} \times C_{i-1} B_{i-1}=A_{i} \times C_{i}\left(C_{i} \times C_{i-1} B_{i-1}\right) \text {, }
$$

and so the map $(*)$ is obtained from the fibration

$$
B_{i} \rightarrow C_{i} \times_{i-1} B_{i-1}
$$

by applying $u_{i}^{*}$, and is therefore a fibration by Lemma 1 of $\$ 4$.

In order to obtain results on inverse limits we will need another axiom.

(I) Every tower of fibrations in $\mathcal{C}$ has an inverse limit, and the functor inv lim: Tow $(\mathcal{C}) \rightarrow \mathcal{C}$ preserves fibrations and aspherical fibrations (and hence weak equivalences by the factorization lemma $(\$ 1)$ ).

Remarks. 1. If $\mathcal{C}$ consists of the fibrant objects of some larger category $\mathcal{C}^{\prime}$, i.e., of those objects $B$ such that $B \rightarrow \dot{e}$ is a fibration, and if fibrations and aspherical fibrations in $\mathcal{C}^{\prime}$ are characterized by lifting properties as in closed model categories [21], then (I) holds (provided that the inverse limits exist in $\mathcal{C}^{\prime}$ ). This fact is what motivated the above definition of fibration in Tow $(\mathcal{C})$.

2. If (I) holds then we easily deduce from Theorem 1 and Remark 2 of $\$ 2$ that the localization functor $\gamma: \mathcal{C} \rightarrow$ Ho $\mathcal{C}$ preserves countable products.

Assume now that (I) holds, let $\left\{A_{i}\right\}$ be an object of Tow $(\mathcal{C})$, and consider the two maps $f, g: \Pi A_{i} \rightrightarrows \Pi A_{i}$, where $f=$ id and $g$ is the map defined "pointwise" by $g\left(\left\{a_{i}\right\}\right)=\left\{p_{i+1}\left(a_{i+1}\right)\right\}$. We form a homotopy equalizer of $f$ and $g$ as in the remark at the end of $\$ 4$, and we observe that there is an obvious map $b$ : $\lim _{i} A_{i} \rightarrow K$.

Le mma. The map $b$ is a weak equivalence.

Proof. Let $\left\{A_{i}^{l}\right\}$ be a path space for $\left\{A_{i}\right\}$. Then we may take $\Pi A_{i}^{I}$ as path space for $\Pi A_{i}$, and it is immediate from the definitions that $K=\lim K_{i}$, where

$$
K_{i}=A_{1}^{I} \times_{A_{1}} \cdots \times_{A_{i-1}} A_{i}^{I}
$$

(assuming, for simplicity of notation, that $A_{i}=e$ for $i \leq 0$ ), and that $b$ is induced by a map of towers $\left\{b_{i}\right\}:\left\{A_{i}\right\} \rightarrow\left\{K_{i}\right\}$, where

$$
b_{i}=\left(s p_{2} \cdots p_{i}, \cdots, s p_{i}, s\right) .
$$

By axiom (I) it is sufficient to prove that each $b_{i}$ is a weak equivalence, and this follows from the fact that $b_{i}$ is right inverse to the aspherical fibration 
$d_{1}{ }^{\circ} \mathrm{pr}_{i}: K_{i} \rightarrow A_{i}$ (which can be factored as a composite of base extensions of maps $d_{1}: A_{k}^{I} \rightarrow A_{k}$ ).

In order to state our theorem, we recall that a map $i: K \rightarrow A$ in an arbitrary category is called a weak equalizer of two maps $f, g: A \rightrightarrows B$ if (a) $f i=g i$ and (b) for any map $i^{\prime}: K^{\prime} \rightarrow A$ such that $f i^{\prime}=g i^{\prime}$ there is a map $j: K^{\prime} \rightarrow K$ (not necessarily unique) such that $i j=i^{\prime}$. The construction in the remark of $\$ 4$ (together with Theorem 1 ) shows that weak equalizers exist in $\mathrm{Ho}_{\mathrm{C}} \mathrm{C}$.

Theorem 4. Let $\mathcal{C}$ be a pointed category of fibrant objects and assume $\mathcal{C}$ satisfies axiom (I). Then for any tower of fibrations $\left\{A_{i}\right\}$ and any group valued functor $T$ on $\mathrm{Ho} C$ which preserves countable products and weak equalizers, there is a natural exact sequence

$$
0 \rightarrow R^{1}\left\lfloor T\left(\Omega A_{i}\right) \rightarrow T\left(A_{\infty}\right) \rightarrow \varliminf_{\lim } T\left(A_{i}\right) \rightarrow 1,\right.
$$

where $A_{\infty}=\lim A_{i}$.

Proof. In view of the remark of $\$ 4$ and the lemma above, we have an exact sequence in $\mathrm{Ho} \mathrm{C}$

$$
\prod \Omega_{A_{i}} \stackrel{\beta}{\rightarrow} \Pi \Omega A_{i} \rightarrow A_{\infty} \rightarrow \prod A_{i} \underset{g}{\stackrel{f}{\rightrightarrows}} \prod_{A_{i}}
$$

The sequence remains exact when $T$ is applied, and the result now follows from the standard computation of $R^{1} \lim$ (cf. [20], for example).

Remark. The first theorem of this type seems to be due to Milnor [20]. We recover his theorem by taking for $\mathcal{C}$ the dual of the category of pointed topological spaces and for $T$ a cohomology functor.

6. A remark on Quillen's axioms. In considering the relation between the axioms of $\$ 1$ and Quillen's axioms for abstract homotopy theory [21] it is natural to ask to what extent the present axioms need to be strengthened in order to become equivalent to Quillen's, or, more generally, to ask for a set of axioms equivalent to Quillen's, but with the emphasis on fibrations and weak equivalences. In order to make the question more precise we will recall some terminology from [21].

Let $\mathcal{C}$ be a category with two distinguished classes of maps, called weak equivalences and fibrations. As usual, a fibration which is also a weak equivalence $w$ ill be called an aspherical fibration. A map $i: A \rightarrow X$ will be said to have the LLP (left lifting property) with respect to a map $p: E \rightarrow B$, and $p$ will be said to have the $R L P$ (right lifting property) with respect to $i$, if for any solid arrow diagram 


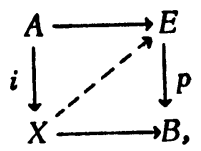

the dotted arrow exists. We call $i$ a cofibration if it has the LLP with respect to all aspherical fibrations.

Consider now the following axioms.

(F) Any map $u$ can be factored $u=s j$ where $j$ is a cofibration and $s$ is a weak equivalence.

(G) Any map $u$ can be factored $u=p i$, where $p$ is a fibration, $i$ is a weak equivalence, and $i$ has the LLP with respect to all fibrations.

Proposition 5. Let $\mathcal{C}$ be as above, and ass ume further that a retract of a weak equivalence (or fibration) is again a weak equivalence (or fibration). If $\mathcal{C}$ satisfies axioms (A), (B), (C), (F), and (G), then $C$ also satisfies all of Quillen's axioms for a closed model category [21], except possibly for the existence of limits.

Proof. By applying (G) to the maps $s$ in axiom (F), we see that $s$ can be assumed to be an aspherical fibration, so both factorization axioms of [21] are satisfied. By applying (G) to any cofibration $u$ which is also a weak equivalence, we easily deduce (cf. the proof of (i) $\Rightarrow$ (iii) in II, $\$ 3$, Lemma 4 of [21]) that such a map is a retract of a map which has the LLP with respect to all fibrations, so $u$ also has this property. Thus the lifting axioms are satisfied, and the other axioms present no difficulty.

Remark. This proposition applies in particular to simplicial sets, with weak equivalence defined by means of the geometric realization functor, and it yields a simple proof of [21, Chapter II, $\S 3$, Theorem 3].

\section{PART II. SHEAVES OF SPECTRA AND GENERALIZED SHEAF COHOMOLOGY}

7. Spectra. In this section we will recall Kan's definition of spectrum and we will outline that portion of the homotopy theory of spectra which will be needed in the rest of this paper. Standard results and definitions from simplicial homotopy theory ([11], [18], [19]; see also [21] and the remark of $\$ 6$ of the present paper) will be used freely, but we will refer to the literature on the homotopy theory of spectra ([14], [17, Appendix], [6]) only for relatively easy results.

A spectrum is a sequence $E=\left\{E_{n}\right\}_{n} \in Z$ of sets with basepoint $*$, together with (basepoint preserving) face operators $d_{i}: E_{n} \rightarrow E_{n-1}$ and degeneracy operators $s_{i}: E_{n} \rightarrow E_{n+1}(i=0,1,2, \cdots)$, such that (a) the usual simplicial identities hold $\left(d_{i} d_{j}=d_{j-1} d_{i}\right.$ for $i<j$, etc.) and (b) each simplex of $E$ has only 
finitely many faces different from *. We define bispectrum similarly, except that the face and degeneracy operators are now indexed by all integers $i$. Bispectra will not be mentioned again until we deal with smash products (Appendix A), but it is to be understood that the definitions and results given for spectra apply with minor modifications to bispectra.

Examples. 1. For each pair of integers $n, k$ with $k \geq 1$, there is a spectrum generated by an $n$ simplex $x$, subject to the relations $d_{i} x=*$ for $i \geq k$. We will generically denote such a spectrum by $\Delta$, we will let $\dot{\Delta}$ be the subspectrum generated by the faces $d_{i} x$, and we will denote by $\Lambda$ any subspectrum of $\dot{\Delta}$ generated by all faces $d_{i} x$ except one.

2. The same definition as in (1) but with $k=0$ defines the $n$-sphere $S^{n}$.

The category of spectra will be denoted $S_{p}$. Its relations with the category $\delta$. of pointed simplicial sets are as follows. There is a functor $S_{p}: \delta_{\text {. }} \rightarrow \delta_{p}$ which "freely adds" the extra degeneracies to a pointed simplicial set. In the terminology of [14], $\mathrm{Sp}_{\mathrm{p}}(K)$ is the spectrum associated to the prespectrum $\{K, S K$, $\left.S^{2} K, \ldots\right\}, S K$ being the suspension of $K$. For any spectrum $E$, we will let $\left\{E_{(q)}\right\}_{q \in Z}$ denote the associated prespectrum, as defined in [14], and with this notation the functor $E \mapsto E_{(0)}$ is right adjoint to Sp. It is also convenient to observe that $E_{(q)}=\left(S^{q} E\right)_{(0)}$, where $S$ is the functor on spectra which raises dimensions by one, i.e., $(S E)_{n+1}=E_{n}$. One should also observe that $E_{(q)}=\Omega\left(E_{(q+1)}\right)$, where $\Omega$ is the ordinary simplicial loop space functor (denoted " $\omega$ " in [14]).

(Warning: $\Omega$ does not preserve weak equivalences and it therefore coincides with the homotopy theoretical loop space functor only on Kan complexes.)

Homotopy groups of spectra are defined in [14] (see also the next paragraph), and a map of spectra is called a weak equivalence if it induces isomorphisms of all homotopy groups. The associated homotopy category will be called the stable bomotopy category and will be denoted $\mathcal{S}_{t a} \mathcal{H}_{0}$. A map of spectra will be called a fibration if it has the RLP (\$6) with respect to all inclusions of the form $\Lambda \hookrightarrow$ $\Delta$ (see Example 1 above). (This is equivalent to Kan's definition [14].) Finally, we will say that $E$ satisfies Kan's extension condition if the map $E \rightarrow *$ is a fibration.

If $E$ satisfies Kan's extension condition, then $\pi_{q} E$ admits the following description: An element of $\pi_{q} E$ is an equivalence class $[x]$, where $x$ is a spherical $q$-simplex of $E$ (i.e., $d_{i} x=*$ for all $i$ ), and $[x]=\left[x^{\prime}\right]$ if and only if there is a $(q+1)$-simplex $b$ such that $d_{0} b=x, d_{1} b=x^{\prime}$, and $d_{i} b=*$ for $i>1$. In view of the existence of the free group functor $F[14]$ with natural weak equivalence $E$ $\rightarrow F(E)$, and in view of the fact that group spectra satisfy the extension condition, this suffices, in principle, to define $\pi_{q} E$ for arbitrary $E$. We also have the following description of $\pi_{q} E$. 
Proposition 6. For any spectrum $E, \pi_{q} E=\left[s^{q}, E\right]$, where $s^{q}$ is the q-sphere defined in Example 2 and where $[\cdot, \cdot]$ denotes maps in Sta. Ha.

Proof. The functor Sp preserves weak equivalences and induces a functor (still to be denoted $S_{p}$ ) $\mathcal{H}_{a} \rightarrow S_{t a} \mathcal{H}_{a}$, where $\mathcal{H}_{a}=H_{0} \delta_{\text {. }}$ is the ordinary (pointed) homotopy category. On the other hand, the functor $E \mapsto F(E)_{(0)}$ from $\delta_{p}$ to $\delta$. preserves weak equivalences and induces a functor $S_{t a} \mathcal{H}_{a} \rightarrow \mathcal{H}_{a}$ which I claim is right adjoint to $\mathrm{Sp}$. To prove this, one proceeds as in the proof of the adjoint functor lemma of $\$ 3$, with the following slight modification: If $a: \mathrm{Sp}_{\mathrm{p}}\left(E_{(0)}\right) \rightarrow E$ and $\beta: K \rightarrow\left(\mathrm{S}_{\mathrm{p}} K\right)_{(0)}$ are the adjunction maps for the original pair of functors

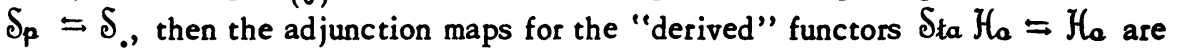
the composites (in $S_{t a} \mathcal{H}_{a}$ and $\mathcal{H}_{a}$ )

$$
\mathrm{Sp}\left(F(E)_{(0)}\right) \stackrel{a}{\rightarrow} F(E) \rightarrow E
$$

and

$$
K \stackrel{\beta}{\rightarrow}\left(\mathrm{Sp}_{\mathrm{p}}\right)_{(0)} \rightarrow(F(\mathrm{Sp} K))_{(0)}
$$

where the second map in (*) is the inverse of the natural map $i: E \rightarrow F(E)$ and that in (**) is induced by $i: \mathrm{Sp} K \rightarrow F(\mathrm{Sp} K)$. It is a straightforward exercise in general nonsense to verify that $(*)$ and $(* *)$ are adjunction maps, and this proves our assertion. The proposition is now obvious: By dimension shifting we may assume $q=0$, and then, since the spectrum $S^{0}$ is obtained from the simplicial zero-sphere (also denoted $S^{0}$ ) by applying $\mathrm{Sp}$, we have $\left[S^{0}, E\right]=\left[\mathrm{Sp}_{\mathrm{p}}\left(S^{0}\right), E\right]=$ $\left[S^{0}, F(E)_{(0)}\right]_{\mathcal{H}_{\mathbf{Q}}}=\pi_{0}\left(F(E)_{(0)}\right)=\pi_{0} F(E)=\pi_{(0)}(E)$. Q.E.D.

Remarks. 1. In the above proof we defined a functor $R T: S_{t a} H_{a} \rightarrow H_{a}$. which we referred to as a "derived" functor of $T$, where $T(E)=E_{(0)}$. This terminology is motivated by the fact that $R T$ is defined by applying $T$ to a "resolution" of $E$ by a spectrum $F(E)$ which satisfies the extension condition. (The point is that $T$ preserves weak equivalences between such spectra.) This terminology also agrees with the technical definition of "right derived functor" introduced in [21].

2. The above proof contains an outline of a proof of a variant of the adjoint functor lemma. We have avoided a precise categorical statement of the variant because there are other variants which we will have occasion to use (some of which require abstract homotopy theory-cf. The orem 3 of I, $\$ 4$, of [21]), and we do not wish to list them all. In the sequel we will refer to any such variant as the "generalized adjoint functor lemma", and leave the details to the reader.

Theorem 5. With the above definitions of fibration and weak equivalence, and with the cofibrations defined to be the injective maps, the category of spectra forms a closed model category in the sense of [21]. 
Proof. We will use the criterion of $\$ 6$. By definition, the fibrations are characterized by the RLP with respect to all $\Lambda \hookrightarrow \Delta$, and it is easy to see (using the corresponding result for simplicial sets) that the aspherical fibrations are characterized by the RLP with respect to all $\dot{\Delta} \hookrightarrow \Delta$. The factorizations required by axioms (F) and (G) can thus be constructed by the small object argument as in [21, II, p. 3.4]. We need only verify that a map obtained by cobase extension from a sum of maps of the form $\Lambda \subset \Delta$ is a weak equivalence. For this we observe that the spectra $\Delta$ can be obtained, up to dimension shift, by applying the functor $\mathrm{Sp}$ to the pointed simplicial set obtained from an ordinary simplex by collapsing its last face to a point. We obtain $\Lambda$ by a similar process, and the desired result now follows from the fact that the cobase extension can be carried out in the category of prespectra [14], where the result can be deduced from the homotopy theory of simplicial sets. Finally, to see that every inclusion is a cofibration in the sense of $\$ 6$, we factor an inclusion $E^{\prime} \hookrightarrow E$ as $E^{\prime} \hookrightarrow E^{\prime} \cup \mathrm{Sp}_{\mathrm{p}}\left(E_{(0)}\right) \hookrightarrow E^{\prime} \cup S^{-1} \mathrm{Sp}_{\mathrm{p}}\left(E_{(1)}\right)$ $\hookrightarrow \cdots \hookrightarrow E$, and we observe that each of these inclusions (up to dimension shift) is a cobase extension of a map obtained by applying $S_{p}$ to a cofibration of simplicial sets, and is therefore a cofibration by an adjoint functor argument.

8. Sheaves of spectra: Local theory. If $X$ is a topological space, a sheaf $E$ on $X$ with values in the category of spectra (or sheaf of spectra) is a contravariant functor

$$
U \mapsto \Gamma(U, E)
$$

from the category of open sets of $X$ to the category of spectra, such that for any open set $U$ and any open cover $\left\{U_{i}\right\}$ of $U$, the sequence

$$
\Gamma(U, E) \rightarrow \prod_{i} \Gamma\left(U_{i}, E\right) \rightrightarrows \prod_{i, j} \Gamma\left(U_{i} \cap U_{i}, E\right)
$$

is exact. (Warning: Because of the finiteness condition in the definition of spectrum, the infinite product of spectra is defined as an appropriate subobjec: of the dimensionwise product.) We will often write $\Gamma(E)$ instead of $\Gamma(X, E)$. We refer to [12], [5] for standard terminology and results concerning sheaves.

If $E$ is a sheaf of spectra, we can regard $E$ as a sequence of sheaves of pointed sets $E_{n}$, with face maps $d_{i}: E_{n} \rightarrow E_{n-1}$ and degeneracy maps $s_{i}: E_{n}$ $\rightarrow E_{n+1}$, such that the usual simplicial identities hold and such that for each $n$,

$$
E_{n}=\bigcup_{N=0}^{\infty} \bigcap_{i>N} \operatorname{ker} d_{i},
$$

this union and intersection being taken in the category of sheaves. This condition can be restated as: Every section of $E_{n}$ over any open set $U$ locally has only 
finitely many nontrivial faces. Another reformulation: For every element $a$ of $E_{n, x}$ (the stalk of $E_{n}$ at $x$ ) one can find a neighborhood $U$ of $x$ and a section $s$ of $E_{n}$ over $U$ such that $s(x)=\alpha$ and $s$ has only finitely many nontrivial faces.

The correspondence between our two definitions is as follows. If $E$ is a sheaf of spectra as in our first definition, we let $E_{n}$ be the sheaf associated to the presheaf $U \mapsto \Gamma(U, E)_{n}$. Conversely, given $E_{n}$ as in the preceding paragraph, we let $\Gamma(U, E)$ be the spectrum whose $n$-simplices are those sections of $E_{n}$ over $U$ with only finitely many nontrivial faces.

Examples. 1. If $E$ is any spectrum and $U$ is an open set of $X$, there is a sheaf of spectra $E_{U}$ which is constant with stalk $E$ on $U$ and whose stalk at any point not in $U$ is the trivial spectrum *.

2. The category of sheaves of abe lian group spectra is equivalent (via the normalization functor) to the category of complexes of abelian sheaves (cf. statement (3) of $\$ 3$ for the unstable analogue). In particular, corresponding to any abelian sheaf $F$ and any integer $q$ there is a stable Eilenberg-Mac Lane sheaf of spectra $K(F, q)$, which by definition is the sheaf of abelian group spectra whose normalization consists of $F$ concentrated in dimension $-q$. (See $\$ 3$ for notational conventions regarding complexes.)

A map between sheaves of spectra will be called a weak equivalence if it induces stalkwise weak equivalences in $\delta_{p}$. Equivalently, if we define the bomotopy sheaf $\pi_{q} E$ to be the sheaf (of abelian groups) associated to the presheaf $U \mapsto \pi_{q} \Gamma(U, E)$, then a map is a weak equivalence if and only if it induces isomorphisms on all homotopy sheaves. We will denote the category of sheaves of spectra by $\delta_{p}(X)$ and the associated homotopy category by $\mathcal{S}_{t a} \mathcal{H}_{0}(X)$.

A map in $S_{p}(X)$ will be called a local fibration if stalkwise it is a fibration in the sense of $\operatorname{Kan}(\$ 7)$. We will denote by $\delta_{p}(X)_{E}$ the full subcategory of $S_{p}(X)$ consisting of the sheaves of spectra which stalkwise satisfy Kan's extension condition.

Proposition 7. The category $\delta_{\beta}(X)_{E}$, with weak equivalence as defined above and with "fibration" defined as "local fibration", satisfies the axioms of $\$ 1$ for a category of fibrant objects. Furthermore, the associated bomotopy category is equivalent to $\delta_{t a} \mathcal{H}_{a}(X)$.

Proof. The only axiom which is not trivial to verify is axiom (D). We omit the proof at this point because the result follows from the proof of Proposition 9 of the next section, where the analogous statement is proved with a stronger notion of fibration. (A direct construction of path spaces is possible using a suitable definition of function spectra, but this involves some unpleasant technical complications.) For the second assertion, we need only note that the free group function $F$ used in $\$ 7$ extends without difficulty to sheaves and induces a functor 
$\mathcal{S}_{t a} \mathcal{H}_{0}(X) \rightarrow \mathrm{Ho}_{\mathrm{o}}\left(\mathcal{S}_{p}(X)_{E}\right)$ quasi-inverse to the obvious functor in the opposite direction.

Proposition 8. The category $\delta_{p}(X)$ satisfies the duals of the axioms of $\$ 1$, with "weak equivalence" being self-dual and with the injective maps playing the role of the class of maps dual to the fibrations.

Proof. Axioms (A), (B), (C), and (E) need only be checked stalkwise, and they are satisfied because of Theorem $5(\$ 7)$. It remains to verify the weak factorization lemma (see the end of $\$ 1$ ). Thus given $u$ : $E \rightarrow E^{\prime}$, let $i: E \rightarrow C E$ be the inclusion of $E$ into its cone (the sheafification of the cone of $[16,6.2]$ ). Then $u$ can be factored as

$$
E \stackrel{(u, i)}{\longrightarrow} E^{\prime} \times C E \stackrel{\mathrm{pr}}{\longrightarrow} E^{\prime}
$$

Corollary 1. The following are equivalent for a map $f$ in $S_{t a} H_{0}(X)$ :

(1) $f$ is an isomorpbism;

(2) $f$ induces stalkwise isomorphisms in Sta. Ha;

(3) $f$ induces isomorphisms on all bomotopy sheaves.

Proof. This is immediate from the fact $(\$ 2)$ that any map in $\mathcal{S}_{t a} \mathcal{H}_{a}(X)$ is of the form $g t^{-1}$, where $g$ and $t$ are maps in $\delta_{p}(X)$.

Corollary 2. The localization functor $\delta_{p}(X) \rightarrow \delta_{t a} H_{a}(X)$ preserves arbitrary sums and finite products. Sta $\mathcal{H}_{a}(X)$ is an additive category.

Proof. We will need the following two facts, which need only be checked when $X$ is a point: (a) an arbitrary sum or a finite product of weak equivalences is again a weak equivalence; and (b) the category of injective weak equivalences under a fixed object has arbitrary sums. [Both assertions about sums follow from the characterization of injective weak equivalences in terms of lifting properties (Theorem 5), and the assertion about products follows from a computation of homotopy groups (which is valid even without the extension condition because geometric realization preserves products).]

Using (a) and (b), the first assertion of the corollary is clear from Theorem 1 and its dual $\left(\oint_{2}\right)$. The additivity of $\delta_{t a} \mathcal{H}_{a}(X)$ is most easily proved by observing that every object can be given a natural group structure (by means of the free group functor, for example), which must then be abelian because by naturality the multiplication map $E \times E \rightarrow E$ is a group homomorphism. Alternatively, one can show that $E \vee E^{\prime} \rightarrow E \times E^{\prime}$ is a weak equivalence, which need only be checked when $X$ is a point, in which case it can be proved by a stable range argument. (See [14, proof of 5.3] for an example of a stable range argument.) This shows 
that every object has a commutative monoid structure, and the existence of inverses can be proved exactly as in the proof that every connected $H$-space has a homotopy inverse [8, Satz 8.2].

We end this section by examining in detail what Theorem $1(\$ 2)$ says about maps in $S_{t a} H_{a}(X)$. We have already mentioned the difficulty of explicitly constructing path spaces in $\delta_{p}(X)$ and thereby getting an explicit definition of homotopy, but as long as we are mapping into a group it is sufficient to have a definition of null homotopy, and for this we can use the standard contractible fibre space $\Lambda G \rightarrow G[15, \S 2]$. Thus we will say that a map $f: E \rightarrow G$ is null-bomotopic if it lifts to $\Lambda G$. An inspection of the definition of $\Lambda G$ shows that $f$ is null-homotopic if and only if there is a degree one map $b: E_{n} \rightarrow G_{n+1}$ such that $d_{0} b=f$, $d_{i} b=b d_{i-1}$ for $i>0$, and $s_{i} b=b s_{i-1}$ for $i>0$. In order to relate this to the definition of homotopy given in $\$ 2$, suppose $f$ is homotopic to * in the sense of $\$ 2$. Then by the homotopy lifting property for the fibration $\Lambda G \rightarrow G$ (cf. the proof of Corollary 1 of Theorem $3(\$ 4)$ ) there is an aspherical fibration $t: E^{\prime} \rightarrow E$ such that $f t$ lifts to $\Lambda G$ and so is null-homotopic.

Corollary 3. Let $E, G$ be sheaves of spectra, with $G$ a group. Then

(i) the natural map

$$
\operatorname{Hom}_{\delta p(X)}(E, G) \rightarrow \operatorname{Hom}_{\delta \operatorname{taH}(X)}(E, G)
$$

is a group bomomorphism whose kernel consists of those maps $f$ such that ft is null-bomotopic for some weak equivalence $t$; and

(ii) any map from $E$ to $G$ in $\mathcal{S}_{t a} \mathcal{H}_{a}(X)$ can be written in the form $f^{-1}$, where $t: E^{\prime} \rightarrow B$ is a weak equivalence and $f: E^{\prime} \rightarrow G$ is a map in $\delta_{p}(X)$.

Proof. The fact that the map is a group homomorphism follows from Corollary 2 by the usual general nonsense. To identify the kernel, suppose $f: E \rightarrow G$ becomes the zero map in $\mathcal{S}_{t a} H_{a}(X)$. If $E$ locally satisfies the extension condition, then the result is immediate from the above remarks, Proposition 7 and Theorem 1. For arbitrary $E$, we apply Theorem 1 (and Remark 2 following it) to $F(f): F(E) \rightarrow$ $F(G)$, and we find that there is an aspherical fibration $t_{1}: Z \rightarrow F(E)$ such that $F(f) \circ t_{1}$ is homotopic to $*$. The base extension of $t_{1}$ by $i: E \rightarrow F(E)$ is then an aspherical fibration $t_{2}: E^{\prime} \rightarrow E$ such that the composite

$$
E^{\prime} \stackrel{t_{2}}{\rightarrow} E \stackrel{f}{\rightarrow} G \stackrel{i}{\rightarrow} F(G)
$$

is homotopic to * and by applying the proof of Proposition 2(b) of $\$ 2$ to the diagram

$$
E_{*}^{\prime \stackrel{f t_{2}}{\longrightarrow}} G \stackrel{i}{\rightarrow} F(G),
$$


we deduce that there is a weak equivalence $t_{3}: E^{n} \rightarrow E^{\prime}$ such that $f t_{2} t_{3}$ is homotopic to *. It is now immediate from the above remarks on null homotopy that $f$ satisfies the condition of (i). Conversely, if $f$ satisfies that condition, then $f$ clearly becomes the zero map in $\mathcal{S}_{t a} \mathcal{H}_{a}(X)$ because $\Lambda G$ is isomorphic to * in $\delta_{t a} \mathcal{H}_{a}(X)$. The proof of (ii) is an even easier application of Theorem 1 and will be omitted.

9. Sheaves of spectra: Global theory. $A$ map $E \rightarrow B$ of sheaves of spectra will be called a global fibration if for each inclusion $U \subset V$ of open sets of $X$, the obvious map

$$
\Gamma(V, E) \rightarrow \Gamma(U, E) \times \Gamma(U, B) \Gamma(V, B)
$$

is a fibration of spectra. We call $E$ flabby (flasque) if the map $E \rightarrow *$ is a global fibration, i.e., if each restriction map $\Gamma(V, E) \rightarrow \Gamma(U, E)$ is a fibration. Note that the global fibrations are the maps with the $\operatorname{RLP}(\$ 6)$ with respect to all maps of the form

$$
\Lambda_{V} U_{\Lambda_{U}} \Delta_{U} \rightarrow \Delta_{V}
$$

(See Example 1 of $\$ 7$ and Example 1 of $\$ 8$ for notation.)

Examples. 1. A sheaf of group spectra $G$ is flabby if and only if its normalization $\left(=\bigcap_{i>0} \operatorname{ker} d_{i}\right)$ is dimensionwise flabby in the usual sense [12]. (This follows from the fact that a homomorphism of group spectra is a fibration if and only if the induced map on normalizations is surjective-see, for example, [21, II, p. 3.8].) In particular, a sheaf of abelian group spectra is flabby if and only if its normalized chain complex is flabby in the usual sense. (Cf. Example 2 of §8.)

2. Using the above characterization of fibrations of group spectra one can imitate the proof of $[12,3.1 .2]$ and prove that a homomorphism of sheaves of group spectra is a global fibration if and only if it is (stalkwise) surjective and its kernel is flabby.

We will now prove that the re exist plenty of global fibrations.

Proposition 9. The category of flabby sheaves of spectra, with the notions of weak equivalence and global fibration, is a category of fibrant objects in the sense of $\$ 1$. Furthermore, axiom $(G)$ of $\$ 6$ bolds in $S_{p}(X)$ relative to the notion of global fibration.

Proof. Axioms (A) and (E) are obvious, $(B)$ and (C) follow by general nonsense from the characterization of global fibrations in terms of lifting properties, 
and (G) (which implies (D)) can be proved using this characterization and a transfinite analogue of the "small object argument" [21, II, p. 3.4]. The point here is that if $X$ is a cardinal such that any open cover of any open set of $X$ has a subcover of cardinality $\leq \kappa$, and if $\alpha$ is the first infinite ordinal bigger than $\kappa$, then the functors $\Gamma(U,-)$ preserve well-ordered direct limits indexed by $\alpha$, so that the objects $\Delta_{U}$ and $\Lambda_{U}$ are small relative to $a$.

In order to state our main result we need one more definition. A sheaf of spectra $E$ is said to be trivial in dimensions greater than $N$ if for each open set $U$ and any two distinct $n$-simplices $u, v$ of $\Gamma(U, E)(n>N)$, one has $d_{i} u \neq d_{i} v$ for at least one $i$. We say that $E$ is bounded below if it is trivial in dimensions greater than $N$ for some $N$. (Note that a group spectrum is bounded below if and only if its normalization consists of the trivial group in sufficiently high dimensions.)

Theorem 6. The global section functor $\Gamma: \delta_{p}(X) \rightarrow \delta_{p}$ preserves weak equivalences of sheaves which are flabby and bounded below.

Proof. Let $E \rightarrow E^{\prime}$ be such a weak equivalence, and let $F$ be the "homotopy fibre", i.e., $F=E \times_{E^{\prime}} \Lambda E^{\prime}$, where $\Lambda E^{\prime}$ is the standard contractible fibre space over $E^{\prime}[15]$. Then from the homotopy exact sequence of the (local) fibration $F \rightarrow E$ (with fibre $\Omega E^{\prime}$ ) we deduce that $\pi_{*} F=0$. Now $F$ is easily checked to be flabby and bounded below, and since $\Gamma F$ is clearly the homotopy fibre of $\Gamma E \rightarrow \Gamma E^{\prime}$, it is sufficient to prove $\pi_{*} \Gamma F=0$. We $w$ ill, in fact, prove by descending induction on $q$ that $\pi_{q} \Gamma(U, F)=0$ for all open $U$. This is certainly true for large $q$ because $F$ is bounded below and $\Gamma(U, F)$ satisfies the extension condition (see the description of the homotopy groups in \$7). Assuming it now for $q+1$, we will prove it for $q$. Let $s$ be a spherical section of $F_{q}$ over $U$. By Zorn's lemma we can find a section $t$ of $F_{q+1}$ defined over some open $U_{0} \subset U$, where $\left(t, U_{0}\right)$ is maximal for the property $d_{0} t=s, d_{i} t=*$ for $i>0$. If $U_{0}=U$ then $[s]=0$ in $\pi_{q} \Gamma(U, F)$ and we are done. If not, let $x$ be any point of $U$ not in $U_{0}$. Since $\pi_{q}(F)=0$, there is a section $t^{\prime}$ over some neighborhood $U_{1}$ of $x$ such that $d_{0} t^{\prime}=s$ and $d_{i} t^{\prime}=*$ for $i>0$. Since $\pi_{q+1} \Gamma\left(U_{0} \cap U_{1}, F\right)=0$ by the induction hypothesis, the sections $t \mid U_{0} \cap U_{1}$ and $t \stackrel{q+1}{q} U_{0} \cap U_{1}$ are homotopic as simplices of $\Gamma\left(U_{0} \cap U_{1}, F\right)$, i.e., there is a section $u$ of $F_{q+2}$ over $U_{0} \cap U_{1}$ such that $d_{0} u=t, d_{1} u=t^{\prime}$, and $d_{i} u=*$ for $i>1$. (To see this, let $\Delta$ be the spectrum generated by a simplex $\sigma$ of dimension $q+2$ with relations $d_{i} \sigma=*$ for $i>1$. Since $\dot{\Delta}$ is equivalent in $S_{t a} H_{a}$. to $S^{q+1}$ (Appendix $A$ ), the obvious map $\dot{\Delta} \rightarrow \Gamma\left(U_{0} \cap U_{1}, F\right)$ is null-homotopic and therefore extends to $\Delta$, $\dot{\Delta} \rightarrow \Delta$ being a cofibration-see Theorem 5.). Now let $\Lambda$ be the subspectrum of $\Delta$ generated by $d_{1} \sigma$. We have a square 


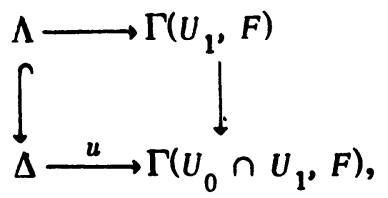

where the unlabelled horizontal arrow takes $d_{1} \sigma$ to $t^{\prime}$. From the definition of fibration we deduce that there is an extension of the homotopy $u$ to a homotopy $\bar{u}$ over $U_{1}$, with $d_{1} \bar{u}=t^{\prime}$. Then $d_{0} \bar{u}$ agrees with $t$ on $U_{0} \cap U_{1^{\prime}}, d_{0} d_{0} \bar{u}=s$, and $d_{i} d_{0} \bar{u}=*$ for $i>0$, so $t$ can be extended to $U_{0} \cup U_{1}$, contradicting its maximality. This completes the proof.

Remarks. 1. The above proof is a homotopy theoretic analogue of the proof of $[12, \mathrm{II}, 3.1 .3]$. It is more complicated than that in [12] because we must take a "homotopy difference" of $t$ and $t$ ' instead of an actual difference. This complication disappears if we work with sheaves of group spectra, in which case the proof in [12] applies without change, using Example 1 above.

2. The boundedness assumption can be removed if $X$ is a noetherian space of finite Krull dimension. The proof of this will appear in the paper cited in the last sentence of the introduction.

3. We can also define a homotopy theoretic analogue of the soft (mou) sheaves of [12], and the analogue of Theorem 6 remains true, provided $X$ is paracompact.

We will end this section with some further results on bounded below flabby sheaves, which will be needed in $\$ \$ 10$ and 11 . We first define the Postnikov decomposition of a spectrum. If $E$ is a spectrum and $N$ is any integer, we will denote by $E[-\infty, N]$ the target of a universal map of $E$ to a spectrum which is trivial in dimensions greater than $N$. (If $E$ satisfies Kan's extension condition, $E[-\infty, N]$ is usually called the $N$ th Postnikov approximation to $E$.) We obtain $E[-\infty, N]$ from $E$ by identifying two simplices of $E$ with the same $N$-dimensional faces. This construction extends to sheaves easily.

Proposition 10. Let $\varrho_{n} \delta_{p} N(X)$ be the category of sheaves of group spectra which are trivial in dimensions greater than $N$. Then $\varrho_{n} S_{p} N(X)$, together with the notions of global fibration and weak equivalence (and cofibration as defined in \$6) is a closed model category in the sense of [21].

Proof. The existence of limits in $\varrho_{n} \delta_{p}{ }^{N}(X)$ presents no difficulties: Projective limits are computed as in $\delta_{p}(X)$ and inductive limits are computed by first computing them in the category of sheaves of group spectra and then applying $[-\infty, N]$. From the fact that the inclusion $\varrho_{n} \delta_{p} N(X) \hookrightarrow \delta_{p}(X)$ has a left adjoint we see that fibrations in $\varrho_{n} S_{p} N(X)$ can be characterized by a lifting property, and thus axiom (G) of $\$ 6$ can be verified by the transfinite small object argument 
as in the proof of Proposition 9. (One needs to use here the well-known homotopy theoretic interpretation of the Postnikov functor.) If we use the criterion of $\$ 6$, the proof will be complete once we verify axiom (F). This can aga in be done by the transfinite small object argument, since it is clear from the following lemma (and the proof of Theorem 5) that aspherical global fibrations in $\mathcal{G}_{n} \delta_{p}{ }^{N}(X)$ are characterized by a lifting property analogous to that for global fibrations.

Lemma. Let $E \rightarrow B$ be an aspherical global fibration in $S_{p}(X)$ whose fibre $F$ is bounded below. Then for each inclusion of open sets $U \subset V$, the map

$$
\Gamma(V, E) \rightarrow \Gamma(U, E) \times_{\Gamma(U, B)} \Gamma(V, B)
$$

is an aspherical fibration of spectra.

Proof. The fibre of the above map is the same as the fibre of $\Gamma(V, F) \rightarrow$ $\Gamma(U, F)$, which is aspherical since both of these spectra are aspherical by Theorem 6 .

Remark., The analogue of Proposition 10 for spectra without group structure is false, even if $X$ is a point. The proof breaks down because the functor $E \mapsto$ $E[-\infty, N]$ does not preserve arbitrary weak equivalences. The analogue for the category $\varrho_{n} \delta_{p}^{+}$of all bounded below spectra also seems false, because the "cell attaching" process required in the proof takes us out of the category $\varrho_{n} \delta_{p}{ }^{+}$ But the analogue for the category of bounded below sheaves of abelian group spectra is true, as we see by working in the equivalent category of bounded below complexes of abelian sheaves.

10. Definition of the cohomology groups. We define a full subcategory of $\mathcal{S}_{p}(X)$ (resp., $\mathcal{S}_{t a} \mathcal{H}_{a}(X)$ ), to be denoted $\mathcal{S}_{p}{ }^{+}(X)$ (resp., $\mathcal{S}_{t a} . \mathcal{H}_{a}{ }^{+}(X)$ ), by the condition $\pi_{q} E=0$ for $q$ sufficiently large. The theory of local fibrations developed in $\$ 8$ for $\delta_{p}(X)$ applies without change to $\delta_{p}{ }^{+}(X)$, and from the resulting description of maps in $\mathrm{Ho}_{\mathrm{p}}{ }^{+}(X)$, we see that $\mathrm{Ho}_{\mathrm{p}}{ }^{+}(X)$ is isomorphic to $\mathcal{S}_{t a} \mathcal{H}_{\mathbf{Q}}{ }^{+}(X)$.

Theorem 7. There is a functor $R \Gamma: \mathcal{S}_{t a} \cdot \mathcal{H}_{a}{ }^{+}(X) \rightarrow \mathcal{S}_{t a} \mathcal{H}_{a}$ such that $R \Gamma(E)$ can be canonically ident ified with $\Gamma\left(E^{\prime}\right)$, where $E \rightarrow E^{\prime}$ is a weak equivalence and $E^{\prime}$ is flabby and bounded below. ( $R \Gamma$ is the right derived functor of $\Gamma$ : $\delta_{p}{ }^{+}(X) \rightarrow \delta_{p}, c f .[21, I, \S 4]$.) Furthermore, $R \Gamma$ commutes with $\Omega(\$ 4)$ and preserves fibration sequences coming from local fibrations.

Definition. The generalized sheaf cobomology group $H^{q}(X, E)$ for $E$ in $\mathcal{S}_{p}{ }^{+}(X)$ is defined by

$$
H^{q}(X, E)=\pi_{-q} R \Gamma(E) .
$$


Proof of Theorem 7. This is more or less clear from the results of $\$ 9$. The details are most easily carried out as follows.

Proposition 10 (in conjuction with Theorem 6 and an easy argument given in $[21, I, \S 4])$ yields the existence of $R \Gamma$ on $\mathrm{Ho}_{2} \oint_{p}{ }^{N}(X)$. We now let $\delta_{p} N(X)$ be defined by $\pi_{q} E=0$ for $q>N$, and we extend $R \Gamma$ to Ho $\delta_{p} N(X)$ by means of the functor $E \mapsto F(E)[-\infty, N]$ from $\mathcal{S}_{p} N(X)$ to $\varrho_{n} \delta_{p}{ }^{N}(X)$. Letting $N$ go to $\infty$ (and using the universal property which defines $\delta_{\text {ta }} \mathcal{H}_{0} .^{+}(X)$ ), we obtain a functor $R \Gamma$ : $\delta_{t a} \mathcal{H}_{a}{ }^{+}(X) \rightarrow \delta_{t a} \mathcal{H}_{a}$. If $E \rightarrow E^{\prime}$ is as in the statement of the theorem and $F\left(E^{\prime}\right)[-\infty, N] \rightarrow G$ is a weak equivalence with $G$ flabby and in $\varrho_{n} \delta_{p} N(X)$, then it is clear from the construction of $R \Gamma$ (cf. [21]) that (for sufficiently large $N$ ) $R \Gamma(E) \approx \Gamma(G)$. We can now apply Theorem 6 to the weak equivalence $E^{\prime} \rightarrow G$, and we find $\Gamma(G) \approx \Gamma\left(E^{\prime}\right)$ in $S_{t a} . H_{0}$, so $R \Gamma(E) \approx \Gamma\left(E^{\prime}\right)$, as required. Finally, $\Gamma$ preserves global fibrations and path spaces of flabby bounded below sheaves, and the last assertion of the theorem follows from this together with the fact that using $\$ 9$ we can convert a local fibration in $\delta_{p}{ }^{+}(X)$ to a global fibration of flabby, bounded below sheaves, without changing the weak homotopy type of the fibre.

Examples. 1. If $E$ is a spectrum, one can define the singular generalized cohomology of $X$ with coefficients in $E$ by applying to the Eilenberg singular complex of $X$ the simplicial analogue of Whitehead's definition [22]. In case $X$ is paracompact and homologically locally connected, it can be shown that this is naturally isomorphic to $H^{*}\left(X, E_{X}\right)$ as defined above, $E_{X}$ being the constant sheaf ( $\$ 8$, Example 1). We omit the proof, since in any case the results of the next section show that our cohomology groups have all the usual properties.

2. If $F$ is an abelian sheaf and $K(F, n)$ is as in Example 2 of $\$ 8$, then $H^{q}(X, K(F, n))$ is the ordinary sheaf cohomology group $H^{q+n}(X, F)$. More generally, if $K^{\circ}$ is a bounded below complex of abelian sheaves and if $E$ is the associated spectrum as in Example 2 of $\$ 8$, then $H^{q}(X, E)=H^{q}\left(X, K^{*}\right)$, the latter group being the usual (hyper-) cohomology group (cf. [13]). This is an immediate consequence of the fact that a flabby resolution $K^{*} \rightarrow I^{*}$ in the usual sense gives rise to a weak equivalence $E \rightarrow E^{\prime}$ ( $E^{\prime}$ being the spectrum associated to $I^{\prime}$ ) such that $E^{\prime}$ is flabby and bounded below in the sense of $\$ 9$. (See $\$ 9$, Example 1. One also needs to observe that $\left.\pi_{q} \Gamma\left(E^{\prime}\right)=H^{q} \Gamma\left(I^{\circ}\right)_{0}\right)$

Complements. 1. If $X$ has finite cohomological dimension and $E$ is a (possibly unbounded) sheaf of spectra which stalkwise satisfies Kan's extension condition, then we define

$$
H^{q}(X, E)=H^{q}(X, E[-\infty, N])
$$

for all sufficiently large $N$, and (as usual) we extend this to arbitrary $E$ by means 
of the free group functor. In order to justify this definition we need to prove that the natural map

$$
H^{q}(X, E[-\infty, N+1]) \stackrel{a}{\rightarrow} H^{q}(X, E[-\infty, N])
$$

is an isomorphism for large $N$. Now it follows from the lemma to be proved in the next section that the fibre of

$$
E[-\infty, N+1] \rightarrow E[-\infty, N]
$$

is isomorphic in Sta. Ha. (X) to $K\left(\pi_{N+1} E, N+1\right)$, and thus we have a fibration sequence in $S_{t a} H_{a}$

$$
R \Gamma\left(K\left(\pi_{N+1} E, N+1\right)\right) \rightarrow R \Gamma(E[-\infty, N+1]) \rightarrow R \Gamma(E[-\infty, N]) .
$$

The homotopy exact sequence of this fibration, in conjunction with Example 2 above, shows that $\alpha$ is an isomorphism for $N \geq p-q, p$ being the cohomological dimension of $X$. (Remark. In case $X$ is a noetherian space of finite Krull dimension, the unbounded case can be handled directly by means of a generalization of Theorem 7. Cf. Remark 2 after Theorem 6, \$9.)

2. Theorems 6 and 7 remain valid if $\Gamma$ is replaced by $\Gamma_{\phi}$ (sections with support in $\Phi$ ), where $\Phi$ is any family of closed subsets of $X$ closed under finite union. We can thus define $H_{\Phi}^{q}(X, E)$ in the obvious way.

3. Theorems 6 and 7 also remain valid if we replace $\Gamma$ by the direct image functor $f_{*}$, where $f: X \rightarrow Y$. We can thus define generalized bigher direct image functors by

$$
R^{q} f_{*}(E)=\pi_{-q} R f_{*}(E)
$$

for $E$ in $\mathcal{S}_{t a} \mathcal{H}_{a}{ }^{+}(X)$. One can check that $R^{q} f_{*}(E)$ is the (abelian) sheaf on $Y$ associated to the presheaf $V \mapsto H^{q}\left(f^{-1} V, E\right)$.

4. The methods of $\$ 3$ can be used to prove an analogue for generalized sheaf cohomology of the hypercovering theorem. Details will be omitted.

5. There is an alternative approach to the definition of generalized sheaf cohomology, which avoids the homotopy theory of sheaves of spectra by using canonical resolutions. The canonical resolution of $E$ is obtained as follows. As in [12] we construct a cosimplicial object in $\delta_{p}(X)$, augmented over $E$, by means of the triple $i_{*} i^{*}$, where $i: X_{\text {dis }} \rightarrow X$ is the natural map, $X_{\text {dis }}$ being the underlying set of $X$ with the discrete topology. To this cosimplicial object we associate a "total" sheaf of spectra $E^{\prime}$ (Appendix B), and we define $H^{q}(X, E)=$ $\pi_{-q} \Gamma\left(E^{\prime}\right)$.

11. Long exact sequences and the fundamental spectral sequence.

Proposition 11. (i) If $\Omega E^{\prime \prime} \rightarrow E^{\prime} \rightarrow E \rightarrow E^{\prime \prime}$ is a fibration sequence in Sta. $\mathcal{H}_{a}{ }^{+}(X)$, then the sequence 


$$
\cdots \rightarrow H^{q-1}\left(X, E^{\prime \prime}\right) \rightarrow H^{q}\left(X, E^{\prime}\right) \rightarrow H^{q}(X, E) \rightarrow H^{q}\left(X, E^{\prime \prime}\right) \rightarrow \cdots
$$

is exact.

(ii) If $U$ is an open set of $X$ then there is a long exact sequence

$$
\cdots \rightarrow H^{q-1}(U, E) \rightarrow H^{q}(X, U ; E) \rightarrow H^{q}(X, E) \rightarrow H^{q}(U, E) \rightarrow \cdots,
$$

where the relative group is by definition the cobomology of $X$ with supports in $X-U$ (see Complement 2 in $\$ 10$ ).

(iii) If $U, V$ are open sets of $X$ and $X=U \cup V$, then the re is a long exact Mayer-Vietoris sequence

$$
\begin{aligned}
\cdots & \rightarrow H^{q-1}(U \cap V, E) \rightarrow H^{q}(X, E) \\
& \rightarrow H^{q}(U, E) \oplus H^{q}(V, E) \rightarrow H^{q}(U \cap V, E) \rightarrow \cdots .
\end{aligned}
$$

Proof. (i) is clear. (ii) is the homotopy sequence of the fibration $\Gamma(X, E) \rightarrow$ $\Gamma(U, E)$, where we have assumed (as we may) that $E$ is flabby and bounded below. (iii) is the homotopy Mayer-Vietoris sequence associated to the cartesian square of fibrations

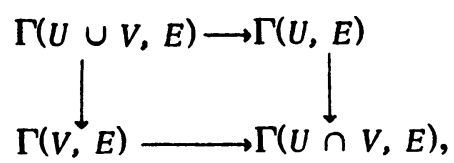

$E$ again being assumed flabby and bounded below.

Remarks. 1. If $X$ is paracompact, then using Remark 3 following Theorem 6 we can obtain long exact sequences analogous to (ii) and (iii) involving closed subsets of $X$.

2. Using the stable Bousfield-Kan spectral sequence (Appendix B) we can generalize (iii) to a spectral sequence for an open cover (or hypercovering) of $X$.

We turn now to the construction of a spectral sequence which is a nonadditive generalization of the hyperhomology spectral sequence and which is a sheaf theoretic generalization of the Atiyah-Hirzebruch spectral sequence.

Lemma. Let $E$ be a sheaf of spectra and suppose that, for some $n, \pi_{q} E=$ 0 for $q \neq n$. Then $E$ is canonically isomorpbic in $S_{t a} . H_{a}(X)$ to the stable Eilenberg-Mac Lane sheaf $K\left(\pi_{n} E, n\right)$.

Proof. Using Example 2 of $\$ 8$ and methods analogous to those of $\S 3$, we see that a map from $E$ to $K\left(\pi_{n} E, n\right)$ in $\delta_{t a} \mathcal{H}_{0}(X)$ is the same as a map from $C_{0}(E)$ to $\pi_{n} E$ in the homotopy category of chain complexes of abelian sheaves (indexed here by subscripts, with differential of degree -1 ), where $\pi_{n} E$ is regarded as a complex concentrated in dimension $n$ and where $C .(E)$ is the normalized chain complex of $E$. Now the group of such maps can be computed by replacing $C_{.}(E)$ by a complex which is zero in dimensions less than $n$ and replacing $\pi_{n} E$ by a 
complex of injectives which is zero in dimensions greater than $n$ and then computing homotopy classes of chain maps. It is trivial to check that this group is Hom $\left(H_{n} C \cdot(E), \pi_{n} E\right)$, and it is clear from the hypothesis on $E$ that a map f: $H_{n} C_{0}(E) \rightarrow \pi_{n} E$ corresponds to an isomorphism $E \rightarrow K\left(\pi_{n} E, n\right)$ if and only if $f$ is an isomorphism. To find such an $f$, we consider the "Hurew icz map" $E \rightarrow Z E$, $\mathrm{Z} E$ be ing the (reduced) free abelian sheaf generated by $E$ (so that $C_{0}(E)$ is the normalization of $\mathbf{Z} E$ ), and observe that by the ordinary Hurewicz theorem applied stalkwise, this map induces an isomorphism on $\pi_{n}$. The existence of an isomorphism $H_{n} C_{0}(E) \approx \pi_{n} E$ now follows from the fact that $H_{n} C_{0}(E)=\pi_{n} Z E$, and this completes the proof.

Theorem 8. For any sheaf of spectra $E$ there is a first and fourth quadrant spectral sequence of cobomological type with $E_{2}^{p q}=H^{p}\left(X, \pi_{-q} E\right)$. If $X$ bas finite cobomological dimension or if $\pi_{q} E=0$ for $q$ sufficiently large, then the spectral sequence converges to $H^{p+q}(X, E)$.

Proof. We may assume that $E$ stalkwise satisfies the extension condition. It follows from the lemma that we have (local) fibrations $K\left(\pi_{n} E, n\right) \rightarrow E[-\infty, n]$ $\rightarrow E[-\infty, n-1]$. By Proposition 11(i), these give rise to long exact sequences in cohomo logy which fit together to form an exact couple and hence a spectral sequence. The identification of the $E_{2}$ term follows from Example 2 of $\$ 10$ and the convergence assertion is trivial.

Corollary (Leray spectral sequence). Let $f: Y \rightarrow X$ be a map and let $E$ be an object of $S_{t a} H_{0}{ }^{+}(Y)$. Then there is a spectral sequence

$$
E_{2}^{p q}=H^{p}\left(X, R^{q} f_{*}(E)\right) \Rightarrow H^{p+q}(Y, E) .
$$

Proof. This is the spectral sequence of The orem 8 applied to $R f_{*}(E)(\S 10$, Complement 3).

Remark. The spectral sequence of Theorem 8 can also be obtained as the Bousfield-Kan spectral sequence (Appendix B) of the cosimplicial spectrum obtained by applying $\Gamma$ to the Godement resolution of $E(\$ 10$, Complement 5$)$.

Finally, we mention that it is possible to define a smash product functor in $S_{t a} \mathcal{H}_{a}(X)$ and to introduce multiplicative structure into generalized sheaf cohomology and into the spectral sequence of Theorem 8. The details are given in Appen$\operatorname{dix} A$.

Appendix A. Smash products in $\mathcal{S}_{t a} H_{a}(X)$. We will need the fact that the spectrum $\dot{\Delta}$ (Example 1 of $\$ 7$ ) is canonically isomorphic in $\delta_{t a} H_{0}$ to a sphere. We can deduce this from the corresponding fact about simplicial sets, as in the treatment of $\Delta$ and $\Lambda$ in the proof of Theorem 5 , or we can simply compute that $\dot{\Delta}$ has the same homology as a sphere. Note that we have a canonical cycle 
$\Sigma(-1)^{i} d_{i} x$ generating the nonzero homology group.

Next, we recall from the homotopy the ory of simplicial sets (or from [21, I, §3]) the explicit description of the boundary homomorphism $\partial: \pi_{n} B \rightarrow \pi_{n-1} F$, where $F \hookrightarrow E \rightarrow B$ is a fibration of spectra. We observe that $\Delta / \Delta$ is a sphere and we represent an element $[s]$ of $\pi_{n} B$ by a map $s: \Delta / \dot{\Delta} \rightarrow B$, which we lift to a map $t: \Delta \rightarrow E$. Then $t \mid \dot{\Delta}: \dot{\Delta} \rightarrow F$ represents $\partial[s]$. This description, together with a suitable version of the homotopy addition theorem (which is proved by ap-plying the Hurewicz theorem to the wedge of spheres obtained from $\dot{\Delta}$ by collapsing all $d_{i} d_{j} x$ to $*$, yields the following.

A1. Let $F \hookrightarrow E \rightarrow B$ be a fibration, let $s$ be a spherical $n$-simplex in $B$, and let $t$ be a lifting of $s$ to $E$ such that all faces $d_{i} t$ are spherical. Then

$$
\partial[s]=\sum(-1)^{i}\left[d_{i} t\right]
$$

in $\pi_{n-1} F$.

We define now the smash product of spectra. Our definition is an adaptation of the definition in [16]. If $E$ and $E^{\prime}$ are spectra, we define their (external) smash product $E \wedge E^{\prime}$ to be the bispectrum (see beginning of $\$ 7$ ) with a $p+q$ simplex $x \wedge x^{\prime}$ for each $p$ simplex $x$ of $E$ and $q$ simplex $x^{\prime}$ of $E^{\prime}$, subject to the identification $* \wedge x^{\prime}=*=x \wedge *$, with faces defined by

$$
d_{i}\left(x \wedge x^{\prime}\right)= \begin{cases}\left(d_{p-i-1} x\right) \wedge x^{\prime} & \text { for }-\infty<i<p \\ x \wedge d_{i-p} x^{\prime} & \text { for } p \leq i<\infty,\end{cases}
$$

and degeneracies defined similarly.

The smash product functor preserves weak equivalences in both variables and induces a functor $\delta_{t a} . H_{a} \times \delta_{t a} . H_{a} \rightarrow$ Ho Bisp (Bisp being the category of bispectra) which can be converted to an internal smash product in $S_{\text {ta }}$. Ha. using the equivalence of $\mathcal{S}_{t a} \mathcal{H}_{a}$ and Ho Bisp established in [16].

A2. Letting $C(E)$ be the unnormalized chain complex of $E$, i.e., the (reduced) free abelian group generated by $E$, with differential $d=\Sigma(-1)^{i} d_{i}$, there is an isomorphism

$$
C(E) \otimes C\left(E^{\prime}\right) \rightarrow C\left(E \wedge E^{\prime}\right)
$$

defined by $x \otimes x^{\prime} \mapsto \epsilon_{p} x \wedge x^{\prime}$ (p= degree of $x$ ), where $\epsilon_{p}$ is defined for all integers $p$ by $\epsilon_{0}=1, \epsilon_{p}=(-1)^{p-1} \epsilon_{p-1}$.

A4. There is a pairing $\pi_{p} E \otimes \pi_{q} E^{\prime} \rightarrow \pi_{p+q}\left(E \wedge E^{\prime}\right)$ defined by $[s] \otimes\left[s^{\prime}\right] \mapsto$ $\epsilon_{p}\left[s \wedge s^{\prime}\right]$, $s$ and $s^{\prime}$ being spherical and $\epsilon_{p}$ being as in $A 2$. The sign is used so that the pairing will be compatible, under the Hurewicz map, with the pairing of homology classes induced by (A3). 
The definition of smash product can be sheafified, and the pairing of A4 induces a pairing of abelian sheaves

$$
\pi_{p} E \otimes \pi_{q} E^{\prime} \rightarrow \pi_{p+q}\left(E \wedge E^{\prime}\right)
$$

for $E$ and $E^{\prime}$ in Sta. Ha. $(X)$.

Suppose, now, that $E, E^{\prime}, E^{\prime \prime}$ are objects of $\mathcal{S}_{t a} \mathcal{H}_{a}{ }^{+}(X)$ and that we are given a map $a: E \wedge E^{\prime} \rightarrow E^{\prime \prime}$. in $\mathcal{S}_{t a}$. Ha $(X)$. (Note that $E \wedge E^{\prime}$ might not be in $S_{t a} H_{a}{ }^{+}(X)$.) Then there is a map

$$
R \Gamma(E) \wedge R \Gamma\left(E^{\prime}\right) \rightarrow R \Gamma\left(E^{\prime \prime}\right),
$$

defined as follows: We may assume that $E$ and $E^{\prime}$ are flabby and bounded below, and using Proposition $8(\$ 8)$ (and the dual of Theorem $1(\$ 2)$ ), we may assume that $\alpha$ is actually a map in $\delta_{p}(X)$. With these assumptions, (AG) is the composite

$$
\Gamma(E) \wedge \Gamma\left(E^{\prime}\right) \rightarrow \Gamma\left(E \wedge E^{\prime}\right) \stackrel{\Gamma(a)^{\prime}}{\longrightarrow} \Gamma\left(E^{\prime \prime}\right) \rightarrow R \Gamma\left(E^{\prime \prime}\right),
$$

the two unlabelled maps being the obvious ones. Using A4 again we obtain from (A6) cup products

$$
H^{p}(X, E) \otimes H^{q}\left(X, E^{\prime}\right) \stackrel{\cup}{\longrightarrow} H^{p+q}\left(X, E^{\prime \prime}\right) .
$$

(If $X$ has finite cohomological dimension, we can eliminate the hypothesis that the sheaves are in $S_{t a} . H_{a}{ }^{+}(X)$ by using Lemma 1 below.)

Example. Let $F$ and $G$ be abelian sheaves and let $E, E^{\prime}, E^{n}$ be $K(F, n)$, $K(G, m)$, and $K(F \otimes G, n+m)$, respectively. It follows from the proof of the lemma of $\$ 11$, together with $A 2$ and $A 4$, that there is a unique pairing $E \wedge E^{\prime} \rightarrow$ $E^{\prime \prime}$ in $\mathcal{S}_{t a} \mathcal{H}_{a}(X)$ such that the corresponding map $\pi_{n}(E) \otimes \pi_{m}\left(E^{\prime}\right) \rightarrow \pi_{n+m}\left(E^{\prime \prime}\right)$ (see $(A 5))$ is the identity map of $F \otimes G$. The resulting cup product agrees with the usual cup product

$$
H^{p+n}(X, F) \otimes H^{q+m}(X, G) \rightarrow H^{p+q+n+m}(X, F \otimes G)
$$

under the identifications of Example 2 of $\$ 10$.

In order to introduce products into the spectral sequence of Theorem 8 , we need to observe that the exact couple defined in the proof of that the orem is actually part of a spectral system (i.e., an $H(p, q)$ system as in [7, Chapter XV]). Thus if we define, for a sheaf $E$ of spectra or bispectra, $E[p, \infty]=$ the fibre of $E \rightarrow E[-\infty, p-1]$, and if we define, for $-\infty \leq p \leq q \leq \infty$,

$$
E[p, q]=(E[p, \infty])[-\infty, q] \text {, }
$$

then the spectral system is given by $H(p, q)=H^{*}(X, E[p, q-1])$, where of course, $E[p, p-1]=*$. The maps $H(p, q) \rightarrow H\left(p^{\prime}, q^{\prime}\right)$ for $\left(p^{\prime}, q^{\prime}\right) \leq(p, q)$, which we will 
denote by $\lambda$, come from the natural maps $E[p, q-1] \rightarrow E\left[p^{\prime}, q^{\prime}-1\right]$; the maps $\delta: H(p, q) \rightarrow H(q, r)$ are the connecting homomorphisms in the long exact cohomology sequences associated to the fibrations $E[q, r-1] \hookrightarrow E[p, r-1] \rightarrow E[p, q-1]$. Note that the exact couple of the proof of Theorem 8 consists of the exact sequences

$$
\cdots \rightarrow H(q-1, q) \rightarrow H(-\infty, q) \rightarrow H(-\infty, q-1) \rightarrow H(q-1, q) \rightarrow \cdots,
$$

but the groups $H(-\infty, q)$ will play no role in our construction of pairings.

Lemma 1. Let $E, E^{\prime}$ be sheaves of spectra, let $p$ and $q$ be (finite) integers, and assume $0 \leq r \leq \infty$. Then

(i) $E[p, \infty] \wedge E^{\prime}[q, \infty] \subset\left(E \wedge E^{\prime}\right)[p+q, \infty]$.

(ii) The inclusions of (i) induce, by passage to the quotients, maps $E[p, p+r] \wedge E^{\prime}[q, q+r] \rightarrow\left(E \wedge E^{\prime}\right)[p+q, p+q+r]$.

Proof. This is simply a matter of checking the definitions.

If we are given a pairing $E \wedge E^{\prime} \rightarrow E^{\prime \prime}$, the pairing (A5) induces (via cup product) a pairing

$$
E_{2}^{p q} \otimes E_{2}^{\prime p^{\prime} q^{\prime}} \rightarrow E_{2}^{\prime p+p^{\prime}, q+q^{\prime}}
$$

of the spectral sequences of $E, E^{\prime}$, and $E^{\prime \prime}$.

Theorem 9. Let $E, E^{\prime}, E^{\prime \prime}$ be sheaves of spectra, and let $E_{r}, E_{r}^{\prime}, E_{\text {, be the }}^{\prime \prime}$ spectral sequences of Theorem 8. Then given any map $E \wedge E^{\prime} \rightarrow E^{\prime \prime}$ in $S_{t a} H_{a}(X)$, the pairing (A8) extends to a pairing of spectral sequences $E_{r} \otimes E_{r}^{\prime}$ $\rightarrow E_{r}^{\prime \prime}$ which on $E_{\infty}$ is compatible with the cup product (A7).

Proof. Let $H, H^{\prime}, H^{\prime \prime}$ be the three spectral systems. Lemma 1 gives us cup products $H(p, p+r) \otimes H^{\prime}(q, q+r) \rightarrow H^{\prime \prime}(p+q, p+q+r)$, which will induce a pairing of spectral sequences provided we verify commutativity of the following (cf. $[10$, IIA]):

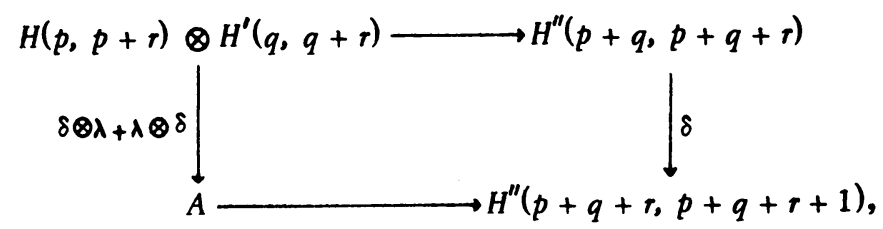

where

$$
A=\left(H(p+r, p+r+1) \otimes H^{\prime}(q, q+1)\right) \oplus\left(H(p, p+1) \otimes H^{\prime}(q+r, q+r+1)\right) .
$$

(Note that according to the sign convention $(\lambda \otimes \delta)\left(u \otimes u^{\prime}\right)=(-1)^{p} \lambda(u) \otimes \delta\left(u^{\prime}\right)$ if $u$ is a cohomology class of dimension p.) This commutativity follows from Lemma 2 below applied to the fibrations 


$$
\begin{aligned}
E[p+r, p+r] & \hookrightarrow E[p, p+r] \longrightarrow E[p, p+r-1], \\
E^{\prime}[q+r, q+r] & \hookrightarrow E^{\prime}[q, q+r] \longrightarrow E^{\prime}[q, q+r-1], \\
E^{\prime \prime}[p+q+r, p+q+r] & \hookrightarrow E^{\prime \prime}[p+q, p+q+r] \rightarrow E^{\prime \prime}[p+q, p+q+r-1] .
\end{aligned}
$$

Note that we have used the dual of Theorem 1 applied to sheaves of bispectra in order to assume that we have a map $E \wedge E^{\prime} \rightarrow E^{\prime \prime}$ in $\Re_{i a p}(X)$.

Lemma 2. Let $p: E \rightarrow B$ and $p^{\prime}: E^{\prime} \rightarrow B^{\prime}$ be local fibrations of sheaves of spectra and let $p^{\prime \prime}: E^{\prime \prime} \rightarrow B^{\prime \prime}$ be a local fibration of sheaves of bispectra. Assume all of the above sheaves are bounded below and locally satisfy Kan's extension condition. Let $i: F \rightarrow E, i^{\prime}: F^{\prime} \rightarrow E^{\prime}$, and $i^{\prime \prime}: F^{\prime \prime} \rightarrow E^{\prime \prime}$ be the fibre inclusions and assume given maps $\alpha: E \wedge E^{\prime} \rightarrow E^{\prime \prime}, \beta: B \wedge B^{\prime} \rightarrow B^{\prime \prime}, \gamma: F \wedge B^{\prime} \rightarrow F^{\prime \prime}$, and $\delta: B \wedge F^{\prime} \rightarrow F^{\prime \prime}$ such that $p^{\prime \prime} \alpha=\beta \circ\left(p \wedge p^{\prime}\right), i^{\prime \prime} \gamma \circ\left(F \wedge p^{\prime}\right)=\alpha \circ\left(i \wedge E^{\prime}\right)$, and $i " \mu \circ\left(p \wedge F^{\prime}\right)=\alpha \circ\left(E \wedge i^{\prime}\right)$. Letting $\partial$ denote the connecting bomomorphism in all three cobomology exact sequences, we bave (using the cup products obtained from $\alpha, \beta, \gamma, \delta$ )

$$
\partial\left(u \cup u^{\prime}\right)=\partial u \cup u^{\prime}+(-1)^{p} u \cup \partial u^{\prime}
$$

in $H^{p+q+1}\left(X, F^{\prime \prime}\right)$, where $u \in H^{p}(X, B)$ and $u^{\prime} \in H^{q}\left(X, B^{\prime}\right)$.

Proof. We will assume first that all sheaves are flabby and all fibrations are global fibrations. An element of $H^{p}(X, B)$ is represented by a section $s$ of the spherical - $p$ simplices of $B$, and an element of $H^{q}\left(X, B^{\prime}\right)$ is represented by a section $s^{\prime}$ of the spherical $-q$ simplices of $B^{\prime}$. Let $t$ be a lifting of $s$ to $E$ such that $d_{i} t=*$ for $i>0$ and let $t^{\prime}$ be a similar lifting of $s^{\prime}$. Then $\partial[s]=$ $\left[d_{0} t\right]$, where $d_{0} t$ is regarded as a section of $F$, and $\partial\left[s^{\prime}\right]=\left[d_{0} t^{\prime}\right]$ (see $A 1$ ). On the other hand, by definition, $[s] \cup\left[s^{\prime}\right]=\epsilon_{-p}\left[s^{\prime \prime}\right]$, where $s^{\prime \prime}$ is the image in $B^{\prime \prime}$ of $s \wedge s^{\prime}$ and $\epsilon_{-p}$ is as in $A 2$. The image $t^{\prime \prime}$ in $E^{\prime \prime}$ of $t \wedge t^{\prime}$ is a lifting of $s^{\prime \prime}$ to $E^{\prime \prime}$, and its nontrivial faces are $d_{-(p+1)^{t}}=\gamma\left(d_{0} t \wedge s^{\prime}\right)$ and $d_{-p} t^{\prime \prime}=\delta\left(s \wedge d_{0} t^{\prime}\right)$. Therefore

$$
\begin{aligned}
\partial\left([s] \cup\left[s^{\prime}\right]\right) & =\epsilon_{-p} \partial\left[s^{\prime \prime}\right]=\epsilon_{-p}\left((-1)^{p+1}\left[d_{-(p+1)} t^{\prime \prime}\right]+(-1)^{p}\left[d_{-p} t^{\prime \prime}\right]\right) \\
& =\epsilon_{-p}(-1)^{p+1} \epsilon_{-p-1} \partial[s] \cup\left[s^{\prime}\right]+\epsilon_{-p}(-1)^{p} \epsilon_{-p}[s] \cup \partial\left[s^{\prime}\right] \\
& =\partial[s] \cup\left[s^{\prime}\right]+(-1)^{p}[s] \cup \partial\left[s^{\prime}\right] .
\end{aligned}
$$

We will now reduce the general case to the special case just considered by showing that we can map the given sheaves to sheaves satisfying the conditions of the above paragraph in a way compatible with all the given data and such that the maps are weak equivalences. By applying the free group functor and then an 
appropriate Postnikov functor to $p$ we can imbed it by a weak equivalence into a map in $\varrho_{n} S_{p} N(X)$ for some $N$, which we can then imbed in a global fibration $\bar{p}$ of flabby sheaves by Proposition 10. Similarly, we imbed $p^{\prime}$ by a weak equivalence into $\overline{p^{\top}}$. Next we can replace $B^{\prime \prime}$ by

$$
B^{n} \cup_{B \wedge B^{\prime}} \bar{B} \wedge \overline{B^{\prime}}
$$

and we can therefore assume that $\beta$ factors as

$$
B \wedge B^{\prime} \rightarrow \bar{B} \wedge \overline{B^{\prime}} \stackrel{\bar{\beta}}{\rightarrow} B^{\prime \prime} .
$$

The rest of the given maps can be summarized by a diagram

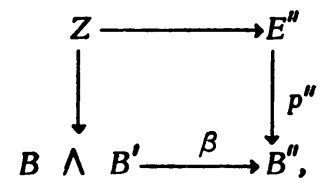

where

$$
Z=\left(B \wedge F^{\prime}\right) \cup_{E \wedge F^{\prime}}\left(E \wedge E^{\prime}\right) \cup_{F \wedge E^{\prime}}\left(F \wedge B^{\prime}\right)
$$

and the unlabelled horizontal and vertical maps are, respectively, $\left(i{ }^{\prime \prime} \delta, a, i^{\prime \prime} \gamma\right)$ and $\left(*, p \wedge p^{\prime}, *\right)$. If we verify that $Z \rightarrow \bar{Z}$ is a stalkwise injective weak equivalence then we can replace $E^{\prime \prime}$ by $\overline{E^{\prime \prime}}=\bar{Z} \cup_{Z} E^{\prime \prime}$ and then convert the resulting map $\overline{E^{n}} \rightarrow B^{\prime \prime}$ into a fibration, and this will complete the reduction. Now one sees by inspection that the map $Z \rightarrow \bar{Z}$ is injective, and to prove it is a weak equivalence we observe that we have a cocartesian square

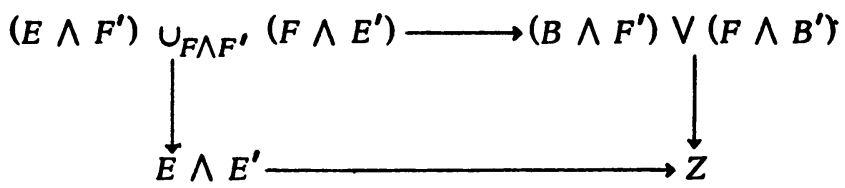

and similarly for $\bar{Z}$, where the vertical arrows are injective. But it follows from $[16,5.5]$ that these squares give rise to Mayer-Vietoris sequences in homotopy, which reduces us to proving a weak equivalence of the spectrum in the upper lefthand corner with its barred analogue, and this aga in follows from the Mayer-Vietoris sequence.

Appendix B. The stable Bousfield-Kan spectral sequence. Let $X$ be a cosimplicial object in the category of spectra, i.e., for each integer $p \geq 0$ we have a spectrum $X^{p}$, and we have coface maps $\delta_{i}: X^{p-1} \rightarrow X^{p}$ and codegeneracy maps 
$\sigma_{i}: X^{p+1} \rightarrow X^{p}, i=0,1, \cdots, p$. We define the total spectrum $T(X)$ as follows: An n-simplex of $T(X)$ is a sequence $\left\{x_{p+n}^{p}\right\}_{p \geq 0}, x_{p+n}^{p} \in X_{p+n}^{p}$, such that

(a) $d_{i} x_{p+n}^{p}=\delta_{i} x_{p+n-1}^{p-1}, 0 \leq i \leq p$, and $s_{i} x_{p+n}^{p}=\sigma_{i} x_{p+n+1}^{p+1}, 0 \leq i \leq p$, (where, for $p=0$, we set $x_{n-1}^{-1}=*$ ); and

(b) $\left\{x_{p+n}^{p}\right\}$ has only finitely many nontrivial faces, these being defined by

$$
d_{i}\left\{x_{p+n}^{p}\right\}=\left\{d_{i+p+1} x_{p+n}^{p}\right\} \text {. }
$$

Replacing " $d$ " by " $s$ " in (B1), we obtain a definition of degeneracies in $T(X)$, which then becomes a spectrum.

We can define approximations $T_{k}(X)$ to $T(X)$ by using finite sequences $\left\{x_{p+n}^{p}\right\}_{p \leq k}$, and we have

$$
T(X)=\underbrace{\lim }_{k} T_{k}(X)
$$

We say $X$ is fibrant if the map of $X^{p+1}$ to the product of $p+1$ copies of $X^{p}$ given by the codegeneracies is a fibration of $X^{p+1}$ onto the "possible image". (The possible image is determined by the cosimplicial identities.) If $X$ is fibrant then the maps $T_{k}(X) \rightarrow T_{k-1}(X)$ are fibrations, and the $E_{1}$ term of the corresponding spectral sequence is the normalization of the cosimplicial graded abelian group $p \mapsto \pi_{*} X^{p}$. Thus the total spectrum and the spectral sequence provide a nonadditive generalization of the spectral sequence of a double complex of abelian groups.

Remark. The total spectrum construction was discovered in connection with the study of function spectra. In fact, if $K$ is a pointed simplicial set and $E$ is a spectrum, we can define a spectrum $K \wedge E$ by formulas similar to those defining the smash product of two spectra. The functor $K \wedge$ - has a right adjoint $\mathcal{H}_{a m}(K,-)$, where $\mathcal{H}_{a m}(K, E)$ is defined as the total spectrum associated to the cosimplicial spectrum $p \mapsto \operatorname{Hom}\left(K_{p}, E\right)$, the latter "Hom" referring to maps of pointed sets. Conversely, the total spectrum $T(X)$ can be defined in terms of function spectra as in $[4, \S 3]$, where the unstable analogue is presented. Note that the situation is better in the stable case, since in the unstable case there is no simple explicit description of the n-simplices of the total object analogous to our definition above.

\section{REFERENCES}

1. M. Artin, Grothendieck topologies, Harvard Seminar Notes, 1962.

2. M. Artin, A. Grothendieck and J.-L. Verdier, Séminaire de géométrie algébrique; Cohomologie étale des schémas, Inst. Hautes Etudes Sci., Paris, 1963/64. 
3. M. Artin and B. Mazur, Étale homotopy, Lecture Notes in Math., no. 100, SpringerVerlag, Berlin and New York, 1969. MR 39 \#6883.

4. A. K. Bousfield and D. M. Kan, Homotopy with respect to a ring, Proc. Sympos. Pure Math., vol. 22, Amer. Math. Soc., Providence, R. I., 1971, Pp. 59-64.

5. G. E. Bredon, Sheaf theory, McGraw-Hill, New York, 1967. MR 36 \#4552.

6. D. Burghelea and A. Deleanu, The homotopy category of spectra. I, II, III, Illinois J. Math. 11 (1967), 454-473; Math Ann. 178 (1968), 131-144; Math. Z. 108 (1969), 154170. MR 36 \#2146; 39 \#3491; \#3492.

7. H. Cartan and S. Eilenberg, Homological algebra, Princeton Univ. Press, Princeton, N. J., 1956. MR 17, 1040.

8. A. Dold, Halbexakte homotopiefunktoren, Lecture Notes in Math., no. 12, SpringerVerlag, Berlin and New York, 1966. MR 33 \#6622.

9.. A. Dold and D. Puppe, Homologie nicht-additiver Funktoren; Anwendungen, Ann. Inst. Fourier (Genoble) 11 (1961), 201-312. MR 27 \#186.

10. A. Douady, La suite spectrale de Adams: Structure multiplicative, Sém. H. Cartan, 11 (1958/59), Exposé 19.

11. P. Gabriel and M. Zisman, Calculus of fractions and homotopy theory, Ergebnisse der Mathematik und ihrer Grenzgebiete, Band 35, Springer-Verlag, New York, 1967. MR 35 \#1019.

12. R. Godement, Topologie algébrique et théorie des faisceaux, Actualités Sci. Indust., no. 1252, Hermann, Paris, 1958. MR 21 \#1583.

13. R. Hartshome, Residues and duality, Lecture Notes in Math., no. 20, SpringerVerlag, Berlin and New York, 1966. MR 36 \#5145.

14. D. M. Kan, Semisimplicial spectra, Illinois J. Math. 7 (1963), 463-478. MR 27 \#2986.

15. - On the k-cochains of a spectrum, Illinois J. Math. 7 (1963), 479-491. MR 27 \#5253.

16. D. M. Kan and G. W. Whitehead, The reduced join of two spectra, Topology 3 (1965), suppl. 2, 239-261. MR 31 \#2720.

17. - Orientability and Poincaré duality in general homology theories, Topology 3 (1965), 231-270. MR 32 \#335.

18. K. Lamotke, Semisimpliziale algebraische Topologie, Die Grundlehren der math. Wissenschaften, Band 147, Springer-Verlag, Berlin and New York, 1968. MR 39 \#6318.

19. J. P. May, Simplicial objects in algebraic topology, Van Nostrand Math. Studies, no. 11, Van Nostrand, Princeton, N. J., 1967. MR 36 \#5942.

20. J. W. Milnor, On axiomatic homology theory, Pacific J. Math. 12 (1962), 337341. MR 28 \# 2544.

21. D. G. Quillen, Homotopical algebra, Lecture Notes in Math., no. 43, SpringerVerlag, Berlin and New York, 1967. MR 36 \#6480.

22. G. W. Whitehead, Generalized homology theories, Trans. Amer. Math. Soc. 102 (1962), 227-283. MR 25 \#573.

DEPARTMENT OF MATHEMATICS, CORNELL UNIVERSITY, ITHACA, NEW YORK 14850 